\title{
Pea Protein Reduce Serum Cholesterol Levels in Hypercholesterolemia Hamsters by Modulating Compositions of Gut Microbiota and Metabolites
}

\section{Li-Tao Tong}

Chinese Academy of Agricultural Sciences

\section{Tianzhen Xiao}

Chinese Academy of Agricultural Sciences

Lili Wang

Chinese Academy of Agricultural Sciences

\section{Cong Lu}

Chinese Academy of Agricultural Sciences

Liya Liu

Chinese Academy of Agricultural Sciences

Xianrong Zhou

Chinese Academy of Agricultural Sciences

\section{Aixia Wang}

Chinese Academy of Agricultural Sciences

\section{Wanyu Qin}

Chinese Academy of Agricultural Sciences

Fengzhong Wang ( $\nabla$ wangfengzhong@sina.com )

Institute of Food Science and Techenology https://orcid.org/0000-0001-7533-0344

\section{Research}

Keywords: pea protein, pork protein, cholesterol, microbiome, metabolome

Posted Date: January 11th, 2021

DOI: https://doi.org/10.21203/rs.3.rs-142744/v1

License: (9) (1) This work is licensed under a Creative Commons Attribution 4.0 International License. Read Full License 


\section{Abstract}

Background: Epidemiological and experimental studies have indicated that an increase of plant proteins in diets, as well as a substitution of red meat proteins or processed meat proteins with plant proteins, was closely associated with the reduction of cardio-cerebrovascular disease (CVD)-related mortality. One of the most important ways of plant proteins to reduce the incidence rate of CVD is to lowering cholesterol concentration in serum and liver. Mounting evidences have attributed this effect of plant proteins to their regulation of gut microbiota. However, it remains to be elucidated whether gut microbiota play decisive roles in modulating host cholesterol by dietary protein from diverse sources of plant or meat. This study attempted to clarify the relevant mechanisms by comparing the difference in cholesterol metabolism modulation between pea and pork proteins intake of hypercholesterolemia hamster, as well as its relationships with gut microbiome and metabolic pathway.

Results: We have verified that there are significant differences in the regulations of serum and liver cholesterol levels among different proteins intake from plants (rice, oat, soybean, pea) or meat (chicken, pork, beef), and the corresponded differences in gut microbiota were also observed. The decisive roles of gut microbiota in regulating host cholesterol were illustrated by the findings that the differences in serum cholesterol level between pea protein and pork protein disappeared in the hamsters treated with antibiotics. The experimental results of cross-over intervention of pea and pork protein showed that the serum cholesterol level was reversed with dietary exchange. Most interestingly, the corresponded changes in abundance of dominant bacteria suggested that the "beneficial" microbe Muribaculaceae were responsible for the inhibitory effect of pea protein on serum cholesterol level, while the opposite effect of pork protein was due to the "harmful" microbe Erysipelotrichaceae. Moreover, dietary pea protein supplement altered cecal metabolites including changes in arginine/histidine pathway, primary bile acid biosynthesis, short chain fatty acids or other lipid-like molecules, which involved in cholesterol metabolism.

Conclusion: Dietary pea protein exerted cholesterol-lowering effects through modulating the growth of selected gut microbiota in hamsters. The substitution of pork protein with pea protein could reshape the gut microbiota, affect the contents of metabolites in caecum, and thus regulate cholesterol metabolism. These findings suggest specific bacteria and metabolites can be potential therapeutic targets of hypercholesterolemia by plant protein intervention.

\section{Background}

Accumulating evidence from epidemiologic and intervention trials indicates that proteins from diverse sources exert distinct different healthy effects on human. Consumption of excessive red meat tends to promote higher risks of mortality compared to other dietary ingredients such as whole grains, fish, poultry, nuts, beans, and low-fat dairy products [1-3]. The latest epidemiological studies indicate that an increase of plant proteins in diets and a substitution of red meat proteins or processed meat proteins with plant proteins are closely associated with the reduction of cardio-cerebrovascula (CVD)-related mortality [4-6]. Of note, lipid disorders characterized by hypercholesterolemia are a non-negligible risk factor for CVD [7, 8]. It has been well established that the consumption of proteins from soybean, pea, rice, oat, and buckwheat could significantly reduce blood cholesterol concentration in human and animal models [9-14]. However, the underlying mechanisms of cholesterol regulation in response to protein from diverse sources like plant and animal remain inconclusive.

Protein from diverse sources, as an elemental but vital macronutrient in the diet, has huge influences on the composition and metabolism of the gut microbiota [15]. The intake of soy protein promotes the growth of family Ruminococcaceae thereby producing more short chain fatty acids (SCFAs) compared to meat proteins. Different proteins lower lipids with diverse degrees which may be the consequence of reshaping the gut microbial community [16]. Indeed, the first bacterium isolated from the rats long ago, a group of microbial cholesterol dehydrogenases encoded by the ismA gene, can convert intestinal cholesterol to coprostanol $[17,18]$. It has been confirmed that gut microbial community structures can be changed by the short-term alterations of diets consisted fully of plant or animal products $[19,20]$. Undoubtedly, the types of dietary protein have important effects on gut microbiota, but there is still a lack of validated evidence for whether plant proteins lower cholesterol through gut microbiota, whether it determines the cholesterol metabolism for a long-term dietary intervention. In addition, plant proteins have common characters in the mechanism of lowering serum cholesterol, that is, they can promote the excretion of bile acids in feces compared to casein and red meat protein, which leads to decreases in bile acids concentrations in enterohepatic circulation. However, the 
effects of different types of protein intake on gut metabolites, their reaction to gut microbiota, and the overall influence on cholesterol metabolisms are rarely reported.

As the increasing demand for high quality protein worldwide, protein from pea easily grows all over the world because of its potential health benefits like lowering low-density lipoprotein cholesterol (LDL-C) and ameliorating gut microbiota balance, which has garnered enormous interest from scientists [21-23]. Meanwhile, one essential meat food in China even the world is pork rich in protein and fat [24]. Therefore, pea and pork proteins were studied to distinguish the lowering-cholesterol mechanism of different types of protein by modulating the gut microbial community. Hamsters were treated with antibiotics to investigate the decisive roles of gut microbiota in the effects of dietary proteins on cholesterol metabolism. The necessary modulation of corresponded gut microbiota induced by different proteins responsible for cholesterol metabolism was further examined by the cross-over intervention of dietary protein either from pea to pork or from pork to pea in hamsters. Microbiota diversity and metabonomics were used to identify the dominant microbiotas and metabolites acting as key modulators of cholesterol metabolism in response to diverse proteins.

\section{Methods}

\section{Preparation of diverse proteins}

Rice, soybean and pea proteins were provided by Anhui Lurong Co., Ltd. (Anhui, China) and Shandong Yantai Dongfang Protein Co., Ltd. (Shandong, China), respectively. Oat powder was provided by Hebei Shijiazhuang Lingfeng Co., Ltd. (Hebei, China), and oat protein was prepared by the alkali-solution and acid-isolation method. Chicken, beef, and pork powder were provided by Shandong Qingdao Derunlong Food Co., Ltd. (Shandong, China), and the animal proteins were prepared by defatting the meat powder with petroleum ether. Briefly, meat powder was stirred with petroleum ether $(\mathrm{m} / \mathrm{v} 1: 4)$ for $1 \mathrm{~h}$ and then the upper organic solvent was removed. The extraction was repeated 3 times to achieve maximum defatting.

\section{Animals and diets}

All animal experimental protocols were approved by the Ethical Committee of Experimental Animal Center of the Institute of Medicinal Plant Development, Chinese Academy of Medical Sciences \& Peking Union Medical College. All the male hamsters $(91.67 \pm 0.29 \mathrm{~g})$ were purchased from Beijing Vital River Laboratory Animal Technology Co., Ltd. and housed in a specific pathogen-free (SPF) animal center. The hamsters were acclimatized at $20.0 \pm 0.5^{\circ} \mathrm{C}$ and $50 \pm 10 \%$ humidity with a $12 \mathrm{~h}$ light-dark cycle and given free access to water and food. The experimental diets were prepared by Beijing Nuokangyuan Biotechnology Co., Ltd. (Beijing, China) according to the diet recommended by the American Institute of Nutrition (AIN)-93G formula with few modifications (Table S1). Proteins in these experimental diets were substituted with the obtained proteins prepared above.

In the protein intervention study, four-week-old hamsters were divided into eight groups $(\mathrm{n}=10)$ based on serum total cholesterol content after 7-day acclimatization and then fed experimental diets for 30 days. One group was conventionally raised with standard diet as control, and the other seven groups were fed with high cholesterol diets containing proteins from rice, oat, soybean, pea, chicken, pork, and beef, respectively.

In the antibiotic (Abx) treatment experiment, twenty hamsters were divided into two groups based on the serum total cholesterol (TC) content and fed with pea or pork protein after 7-day acclimatization. Two phyla Firmicutes and Bacteroidetes belong to Bacteria were manifested to be the majority of mammal's gut microbiota [25]. Vancomycin hydrochloride and Bacitracin Zinc have strong sterile effects on Firmicutes and Bacteroidetes while were hardly absorbed by the host gastrointestinal tract [26]. The hamsters were administered drinking water containing Vancomycin hydrochloride $(0.5 \mathrm{~g} / \mathrm{L}$; Shanghai yuanye Bio-Technology Co., Ltd., Shanghai, China) and Bacitracin Zinc ( $1.0 \mathrm{~g} / \mathrm{L}$; Shanghai yuanye Bio-Technology Co., Ltd.) for 30 days. The lipid profiles and the gut microbiota were analyzed before (Pea_Abx_0d, Pork_Abx_0d) and after (Pea_Abx_30d, Pork_Abx_30d) the feeding with Abx treatment.

In the cross-over dietary protein experiment, there were two groups each containing 20 hamsters respectively feeding pea and pork protein during the first month. After 30 days of feeding, fasting blood was collected and the pea group was then divided into two 
groups based on their serum TC concentration for further feeding pea (pea) and pork protein (Pea_Pork), respectively. Likewise, the pork group was divided into two groups for further feeding pea (Pork_Pea) and pork protein (pork), respectively.

The daily food intake of hamsters was monitored to ensure no difference in the contents of protein, starch and lipid among all experiments, and the body weight was recorded once a week. Hamsters were fasted overnight before being sacrificed at the end of these experiments. Blood was collected from the orbital plexus by diethyl ether anesthetization, and centrifuged at $4^{\circ} \mathrm{C}, 3500 \mathrm{rpm}$ for 10 minutes. The liver, caecum, colon and fresh feces were immediately collected and placed on liquid nitrogen. All the tissues were stored at $-80^{\circ} \mathrm{C}$ for further analysis.

\section{Biochemical analysis}

The lipid profiles, including serum TC, high-density lipoprotein cholesterol (HDL-C), LDL-C, and triglyceride (TG), were detected by Fully Automatic Biochemical Analyzer (Hitachi, Tokyo, Japan). The apolipoprotein A, B, E contents in serum; the TC, TG and free cholesterol (FC) contents in liver; the TC and total bile acid contents in feces were measured using Elisa kits from Applygen Technologies Co., Ltd. (Beijing, China). The liver cholesterol ester (CE) was calculated by liver TC minus liver FC. The concentrations of 3-hydroxy-3-methyl glutaryl coenzyme A reductase (HMG-CoA reductase), cholesterol 7a-hydroxylase (CYP7A1), fatty acid synthase (FAS), cholesterol acyltransferase (ACAT), lipoprotein lipase (LPL), and low density lipoprotein receptor (LDLR) in liver were measured with Elisa Kits (Shanghai Enzyme-linked Biotechnology Co., Ltd. Shanghai, China) following manufacturer's instructions.

\section{SCFA analysis}

Total SCFAs were extracted from fecal samples $(10 \mathrm{mg})$ supplemented in $10 \mu \mathrm{l}$ of internal standards $(0.0125 \mu \mathrm{l} / \mu \mathrm{l}$ 2-ethylbutyric acid, Sigma-Aldrich) and $500 \mu \mathrm{l}$ of methanol (Majorbio Bio-Pharm Technology Co., Ltd., Shanghai, China). The extracts were detected using a gas chromatographic coupled with a mass spectrometer (Agilent Technologies Inc, CA, USA), and quantified by the Masshunter quantitative software. SCFAs standards were mixtures of acetate, propionate, butyrate, isobutyrate, valerate, hexanoate, isohexanoate (Darmstadt, Germany), and isovalerate (Sigma-Aldrich).

\section{Real-time quantitative PCR}

Total RNA from liver tissues was extracted using TRNzol Reagent (Tiangen Biotech Co. Ltd., Beijing) and measured with a NanoDrop spectrophotometer (Therno scientific). Reverse transcription was performed using the PrimeScript RT reagent Kit with gDNA Eraser (Takara, Beijing, China) and $1 \mu \mathrm{g}$ of total RNA in ABI7500 Real-Time PCR instrument. The qPCR primers were designed and synthesized (Invitrogen, Beijing, China) as shown in Table S2. The quantitative PCR reaction was performed using SYBR Green Premix (Takara, Beijing, China) reagents and run in Real-Time PCR Instrument (Applied Biosystems, USA). Then, the relative expression of target gene was analyzed using the $2^{-\triangle \Delta C T}$ method.

\section{Gut microbiota analysis}

Genomic DNA was extracted from fecal samples using the E.Z.N.A. ® Stool DNA Kit (Omega Bio-tek, Norcross, GA, U.S.). The quality of extracted DNA was checked by $1 \%$ agarose gel, and DNA concentration and purity were determined by NanoDrop 2000 UV-vis spectrophotometer (Thermo Scientific, Wilmington, USA).

The V3-V4 hypervariable regions of the 16S rRNA gene were subjected to high-throughput sequencing by Beijing Allwegene Tech, Ltd (Beijing, China) with the Illumina Miseq PE300 sequencing platform (Illumina, Inc., CA, USA). The V3-V4 region of the bacteria 16S rRNA gene was amplified with the universal primers of the forward $338 \mathrm{~F}$ (5'-ACTCCTACGGGAGGCAGCAG-3) and the reverse 806 R (5'-GACTACHVGGGTWTCTAAT-3') by an ABI GeneAmp® 9700 PCR thermocycler (ABI, CA, USA). These primers contained a set of 8-nucleotide barcodes sequences unique to each sample. The PCR amplification of 16S rRNA gene was performed as follows: initial denaturation at $95^{\circ} \mathrm{C}$ for $3 \mathrm{~min}, 27$ cycles of denaturing at $95^{\circ} \mathrm{C}$ for $30 \mathrm{~s}$, annealing at $55^{\circ} \mathrm{C}$ for $30 \mathrm{~s}$ and extension at $72^{\circ} \mathrm{C}$ for $45 \mathrm{~s}$, single extension at $72^{\circ} \mathrm{C}$ for $10 \mathrm{~min}$, and end at $10^{\circ} \mathrm{C}$. The PCR mixtures contain $5 \times$ TransStart FastPfu buffer $4 \mu \mathrm{L}$, $2.5 \mathrm{mM}$ dNTPs $2 \mu \mathrm{L}$, forward primer $(5 \mu \mathrm{M}) 0.8 \mu \mathrm{L}$, reverse primer $(5 \mu \mathrm{M}) 0.8 \mu \mathrm{L}$, TransStart FastPfu DNA Polymerase $0.4 \mu \mathrm{L}$, template DNA $10 \mathrm{ng}$, and finally ddH2O up to $20 \mu \mathrm{L}$. Amplicons were extracted from $2 \%$ agarose gels, purified using the AxyPrep DNA Gel Extraction Kit (Axygen Biosciences, Union City, CA, USA), and quantified using QuantiFluor ${ }^{\text {TM }}$-ST (Promega, USA). Purified 
amplicons were pooled in equimolar and paired-end sequenced $(2 \times 300)$ on an Illumina MiSeq platform (Illumina, San Diego, USA).

The raw 16S rRNA gene sequencing reads were demultiplexed, quality-filtered by fastp version 0.20 .0 and merged by FLASH. In brief, quality control criteria mainly included removal of low-quality sequences and barcode sequence: the reads at any site receiving an average quality score of $<20$ within the $50 \mathrm{bp}$ sliding window set to reduce sequencing error, the truncated reads shorter than $50 \mathrm{bp}$, reads containing ambiguous characters. Moreover, only overlapping sequences longer than $10 \mathrm{bp}$ were allowed to assemble and the maximum mismatch ratio of the overlap region is 0.2 . Samples were distinguished according to the barcode and primers, and the sequence direction was adjusted. There was 2 nucleotide mismatch allowed in primer matching while no allowance in barcode mismatching. The acquired high-quality sequences were classified into operational taxonomic units (OTU) at $97 \%$ identity using UPARSE version 7.1. Chimeric sequences were identified and removed. The taxonomy of each OTU representative sequence was analyzed by RDP Classifier version 2.2 against the $16 \mathrm{~S}$ rRNA Silva v138 database using a confidence threshold of 0.7. After these progresses, all sequences were picked randomly for further analyses according to the least number to eliminate the differences caused by sequencing depth among diverse samples.

\section{Untargeted LC-MS metabolomics}

The metabolites were extracted from caecum contents using a 400 methanol: water (4:1,v/v) solution. The mixture was allowed to settle at $-20^{\circ} \mathrm{C}$ and treated by High throughput tissue crusher Wonbio-96c (Shanghai wanbo biotechnology co., LTD) at $50 \mathrm{~Hz}$ for 6 min, then followed by vortex for $30 \mathrm{~s}$ and ultrasonication at $40 \mathrm{kHz}$ for $30 \mathrm{~min}$ at $5^{\circ} \mathrm{C}$. The samples were placed at $-20^{\circ} \mathrm{C}$ for $30 \mathrm{~min}$ to precipitate proteins. After centrifugation at $13000 \mathrm{~g}$ at $4^{\circ} \mathrm{C}$ for $15 \mathrm{~min}$, the supernatant was carefully transferred to sample vials for LC-MS/MS analysis. Equal volumes of all samples were mixed for preparing pooled quality control (QC) samples.

Chromatographic separation of the metabolites was performed on an ExionLCTMAD system (AB Sciex, USA) equipped with an ACQUITY UPLC system with UPLC HSS T3 column (100 mm × $2.1 \mathrm{~mm}$ i.d., $1.8 \mu \mathrm{m}$; Waters, Milford, USA), and a quadrupole time-offlight mass spectrometer (Triple TOFTM5600+, AB Sciex, USA) with an electrospray ionization (ESI) source. The raw data was imported into the Progenesis QI 2.3 (Nonlinear Dynamics, Waters, USA) for peak detection and alignment. Metabolic features detected at least $80 \%$ in any set of samples were retained. Statistical analysis was performed on log transformed data for normalization and imputation to identify significant differences in metabolite levels between comparable groups. All of the metabolite variables were scaled to pareto Scaling prior to orthogonal partial least squares discriminant analysis (OPLS-DA). The OPLS-DA model validity was evaluated from model parameters $\mathrm{R}^{2}$ and $\mathrm{Q}^{2}$, which provided information for the interpretability and predictability, respectively, to avoid the risk of over-fitting of the model. Variable importance in the projection (VIP) was calculated in OPLS-DA model. P values were estimated with paired Student's t-test on Single dimensional statistical analysis.

\section{Statistical analysis}

The differences in the concentrations of lipids in serum, liver, fecal, the contents of enzymes related to lipid metabolism in liver, the contents of SCFAs and bile acid in fecal, and relative abundance of bacteria among the eight groups were evaluated using one-way analysis of variance (ANOVA) by SPSS 22.0 software. Tukey-Kramer's multiple comparison post hoc test was also used to express significant differences among the eight groups. P value less than 0.05 was declared significantly. Community richness was evaluated by Simpson, Ace, Chao and Shannon. Bray Curtis similarity clustering analysis was performed by R package (version R 3.0.2). Multiple group comparisons were conducted by Kruskal-Wallis $\mathrm{H}$ test and two-group comparisons were performed by Wilcoxon ran-sum test. Linear discriminant analysis (LDA) coupled with effect size measurements (LEfSe) was performed to discover highly-dimensional gut bacteria and characterize the differences between two or more biological conditions.

\section{Results}

\section{Diverse proteins modulated serum and liver cholesterol levels in various degrees}

Compared with the initial body weights of hamsters before feeding, a marked increase in body weight for all groups after the daily proteins intake was observed, but there was no significant difference among each group except for the beef protein group which 
exhibited the most body weight gain of $46.5 \pm 3.1 \mathrm{~g}(P<0.05)$ (Table S4). There was no significant difference in the liver weight among all groups $(P>0.05)$.

It was clearly shown that the serum and liver lipids profiles of plant groups were significantly different from those of meat groups after 30 days of feeding with diverse proteins (Fig. 1). Hamsters fed with plant proteins presented lower contents of TC (Fig. 1a, f) and TG (Fig. 1d, i) in serum and liver than the ones fed with red meat proteins including pork and beef $(P<0.05)$, as same as serum LDL-C levels (Fig. 1b). Of note, as one kind of meat protein, chicken protein had significantly lower serum TC and LDL-C levels than red meat proteins, but not bean proteins (soybean and pea). Oat protein displayed the best cholesterol-lowering effect revealed by its lowest serum TC and LDL-C levels of all proteins. Serum HDL-C contents of pea and meat groups were slightly higher than those of the other plant groups without significance, but significantly higher than that of control group. Intervention with plant proteins reduced the Al index and liver $\mathrm{CE}$ significantly compared to meat proteins. The similar trend of liver lipid profiles was observed that the red meat groups exerted the highest levels of liver TC, TG and CE, followed by chicken, while plant showed the lowest levels $(P$ $<0.05)$.

The effects of diverse proteins on lipids secretion (Fig. S1a-d) and serum levels of apolipoproteins (Apo) (Fig. S1e-h) were also detected. Fecal weights of hamsters fed with oat, pea and beef proteins were significantly higher than those of the other groups. Plant and chicken proteins significantly increased fecal TC compared to red meat proteins. Moreover, plant proteins significantly promoted the fecal total lipids and bile acid than animal proteins. The significantly higher ApoE contents were found in all groups compared to control, and beef group showed the highest content. The ApoA levels of plant groups were significantly higher than those of meat groups, and the ApoB content was significantly decreased only induced by pork protein. There was no significant difference in ApoA/ApoB between control, oat, rice and soybean groups which was significantly lower than meat groups $(P<0.05)$.

The effects of diverse proteins on related enzymes of liver lipids metabolism also showed the same significant difference between plant and meat proteins (Fig. S2). The levels of liver HMG-CoA reductase in plant groups were lower than that of meat groups in which pork group showed much higher level than the other groups (Fig. S2a). The concentrations of liver CYP7A1 were significantly enhanced by plant groups while reduced by pork group (Fig. S2b). No significant difference in FAS concentration was found among control, soybean and chicken groups, while a considerable decline in pea and a slight increase in pork and beef groups (Fig. S2c). Interestingly, among plant groups, the content of ACAT was the highest in soybean group, and the contents of LDLR and LPL were the highest in oat group (Fig. S2d-f). Metabolic gene expression (Liver X Receptor: LXR, Farnesoid X Receptor: FXR; and Sterol Regulatory Element Binding Protein-2: SREBP-2) in the liver indicated that consumption of protein decreased the expression of SREBP-2 and LXR mRNA compared to control. Only pork protein significantly increased SREBP-2 expression to the extent which was over 2-fold higher than soybean (Fig. S2g-i) $(P<0.05)$.

\section{Diverse proteins altered gut microbiota correspondly}

According to the values obtained from Shannon and Simpson indices (Fig. S3a, b), oat and pea groups displayed the highest diversity which showed the most significant difference from the meat groups $(P<0.001)$, although rice and soybean also showed higher diversity compared to meat groups at the significance of $P<0.05$ and $P<0.01$, respectively. As for Chao and Ace indices implying the evenness of microbial community (Fig. S3c, d), there were significantly lower values in beef group compared to other groups among which no statistical difference was found. The distance of principal coordinates analysis (PCA) and principal coordinate analysis (PCoA) based on weighted unifrac plots displaying partitions by group was visually representative of similarity among all samples (Fig. S3e, f). Pea and oat groups were clearly separated from the other groups, while meat groups were clustered closely, suggesting that hamsters fed with meat proteins had similar microbiota community structures which was such different from pea and oat proteins. Similar trend was displayed in the hierarchical clustering tree at OTU level which showed that oat and pea as one subgroup was such different from the others; control and rice groups, soybean group as two different subgroups both were clustered away from meat subgroup (Fig. S3g). The hierarchical clustering analysis disclosed apparent separation of plant from meat groups which was in agreement with the results of PCA and PCoA. These findings demonstrated that the composition of gut bacteria exhibited profoundly diverse responses to proteins from different sources $(P<0.05)$.

At the phylum level, Firmicutes and Bacteroidetes were the two predominant phyla in all eight groups, accounting for $61.33 \%-86.84 \%$ and $12.27 \%-36.32 \%$ of the total OTU, respectively. Hamsters fed with meat proteins had higher relative abundance of Firmicutes but lower Bacteroidetes than those fed with plant proteins. The most accumulated relative abundance of Firmicutes 
was observed in beef group while the lowest one was present in oat group (Fig. 2a). Correspondingly, oat group performed the highest ratio of Bacteroidetes to Firmicutes (B/F), followed by rice group without significant difference from control, soybean and pea groups, while pork and beef groups showed the lowest ratio (Fig. 2b). The predominant genus were norank_f_Eubacteriaceae, norank_f_Erysipelotrichaceae, and norank_f_Muribaculaceae. Meat groups shared great similarity in microbial community structures with the highest relative abundance of norank_f_Eubacteriaceae $(40.75 \% \pm 0.84 \%)$, followed by norank_f_Erysipelotrichaceae (25.85\% $\pm 2.89 \%)$, and norank_f_Muribaculaceae $(13.16 \% \pm 1.28 \%)$ compared to plant groups which displayed higher microbial richness (Fig. 2c). Pea group presented four genus accounting for $10.43 \%, 14.81 \%, 16.78 \%$ and $17.35 \%$ of relative abundance, respectively, followed by four approximate genus accounting for $4.57 \%, 4.67 \%, 4.77 \%$ and $5.75 \%$, respectively. This data was in agreement with the results presented in alpha diversity (Fig. S3). Comparisons of pea and pork showed the significantly discrepant bacteria at phylum and family level (Fig. 2d). Their significant difference was not only presented in the dominant phyla Firmicutes and Bacteroidetes, but also in the top 6 family including Eubacteriaceae, Erysipelotrichaceae, Muribaculaceae, Ruminococcaceae, Lachnospirace, and Oscillospiraceae. Different gut microbiota compositions at family level were subsequently characterized in diverse proteins groups (Fig. 2e-I). All groups were dominated by the top three microbiotas including Eubacteriaceae, Erysipelotrichaceae and Muribaculaceae, except for pea group which evenly enriched in Erysipelotrichaceae, Ruminococcaceae, Eubacteriaceae and Muribaculaceae (Fig. 2i). The relative abundances of Erysipelotrichaceae was the highest of all microbiota in control group (35.96\%) (Fig. 2e), as well as in rice group (35.42\%) (Fig. 2f), but that of Muribaculaceae was the highest in oat group (Fig. 2g). Compared to control group, both oat and pea groups led to an increase in Muribaculaceae and a decrease in Eubacteriaceae and Erysipelotrichaceae $(P<0.05)$. Soybean and meat groups presented similar relative abundance of Eubacteriaceae but different ratio of Erysipelotrichaceae/Muribaculaceae, in which soybean (1.40) and chicken (1.78) groups showed much lower $(P<0.05)$ ratio than red meat groups (2.65 for pork; 3.50 for pork) (Fig. 2h, j-l).

The important bacterial taxa contributing to the discrepancies produced by diverse proteins supplements were depicted in LEfSe plots (Fig. 3a). Most taxa selected by LEfSe analysis were enriched in oat and pea groups, but not in control, soybean and pork groups. Among 4 taxa in meat groups and 23 taxa in plant groups, Bacteroidota and Firmicutes at phylum level were significantly enriched in oat and beef group, respectively. This data was consistent with the findings mentioned above that at phylum level Bacteroidota were significantly enriched in oat groups while Firmicutes were mainly in beef (Fig. 2a). The Muribaculaceae family predominated by genera norank_f_Muribaculaceae were enriched in oat group which were of great importance revealed by LDA value $>4.5$. The Oscillospirales order predominated by Ruminococcaceae family were enriched in pea groups exerting great significance revealed by LDA value > 4.5. Within Bacteroidota phylum, three genera Prevotellaceae_UCG_001, Prevotellaceae_NK3B31_group and norank_f_Muribaculaceae were enriched in oat group; two genera Bacteroides and Alloprevotella were enriched in pea group and one genus Alistipes was enriched in chicken group (Fig. 3b). Within Firmicutes phylum, the genus norank_f_Erysipelotrichaceae were enriched in beef group; two orders Oscillospirales and Clostridia_UCG_014 were enriched in pea groups, two genera Eubacterium_ruminantium_group and Family_XIII_UCG-014 were enriched in oat group and one genus lleibacterium were enriched in rice group. The enrichment of Rikenellaceae at family level was mainly attributed to the abundant genus Alistipes. The interconnection networks illustrated the co-occurrence patterns of bacterial community at family level in diverse groups (Fig. 3c). Desulforibrionaceae, Atobobiaceae and Bifidobacteriaceae were unique in control, rice and soybean group, respectively. Prevotellaceae were in both pea and oat groups, and Rikenellaceae were in oat and chicken groups.

As the main metabolites of gut microbiota and their direct effects on lipid profiles, fecal SCFAs content of pork group was the lowest, but those of soybean and oat groups were highest, followed by pea and chicken groups without significant difference from control, rice and beef groups (Fig. 3d. $P<0.05$ ). Redundancy analysis (RDA) was conducted to clarify the correlation between diverse proteins and SCFAs or serum lipid profiles (Fig. 3e, f). Propionate contributed the differences generated by diverse proteins much more than acetate, bile acid, and butyrate (Fig. 3e). Rice and pea groups were positively correlated with acetate, propionate and butyrate, while the meat groups displayed the converse trend. The positive association of soybean group with acetate and rice group with butyrate were observed, but not meat groups. Pea and oat groups exhibited negative association with serum TC and LDL-C while meat groups presented the opposite correlation (Fig. 3f). Overall, plant proteins performed better effects on lowering cholesterol with complex changes in the gut microbiota together with the promoted generation of the SCFAs metabolites compared to meat proteins. 
The relationship between serum lipids, amino acid compositions (Table S3) and gut microbiota were analyzed by the Spearman to explore the underlying mechanism of gut microbiota affecting host cholesterol with diverse proteins (Fig. 3g-i).

Norank_f_Eubacteriaceae, norank_f_Erysipelotrichaceae and unclassified_f_Erysipelotrichaceae clustered as Branch 1 were inversely associated with the amino acids, which were different from other genera. Likewise, the amino acid compositions were divided into two clusters based on the converse correlation with the gut microbiota. One was the left set of amino acids displaying negative correlation with Branch 1 including phenylanine (Phe), glycine (Gly), proline (Pro), tyrosine (Tyr), arginine (Arg), serine (Ser), glutamine (Glu), cystine (Cys) and Valine (Val); the other was the right set showing positive correlation with Branch 1 including alanine (Ala), methionine (Met), leucine (Leu), threonine (Thr), histidine (His), lysine (Lys) asparagine (Asp) and isoleucine (Ile). Val and Cys displayed negative association with norank_f_Eubacteriaceae $(\mathrm{R}=-0.67,-0.68, P<0.001)$, while Ser and His showed positive association with norank_f_Erysipelotrichaceae and Ileibacterium $(R=0.79,0.76, P<0.001)$, respectively (Fig. $3 g)$. The correlation heat map revealed that host lipid profiles were extensively associated with the gut microbiota (Fig. 3h). Branch1 were negatively associated with fecal TC, bile acid, while positively correlated with liver lipids and serum TC, LDL-C, as well as the positive association of norank_f_Erysipelotrichaceae with fecal lipids and liver TG $(\mathrm{R}=0.61,0.60, P<0.001)$.

Norank_f_Muribaculaceae and Eubacterium_ruminantium_group displayed inverse association with serum and liver lipid profiles, especially serum LDL-C $(R=-0.62,-0.54 P<0.001)$. SCFAs are important metabolites of gut microbiota including acerate, butyrate, and propionate (Fig. $3 \mathrm{i}$ ). Butyrate, valerate and isovalerate were clustered into the first branch and propionate, while acetate and isobutyrate were clustered into the second branch. The first branch were positively correlated with Alistipes while negatively correlated with Allobaculum. Ruminococcous and unclassfied_f_Lachnospiraceae were positively associated with acetate $(\mathrm{R}=$ $0.56,0.57 P<0.001)$, and isobutyrate $(R=0.62,0.57 P<0.001)$. Branch1 were negatively associated with all SCFAs which showed positive correlation with norank_f_Muribaculaceae and Eubacterium_ruminantium_group.

\section{Antibiotic treatment abolished the effects of proteins on serum cholesterol modulation}

To investigate the decisive roles of gut microbiota in regulating cholesterol by proteins, the hamsters were treated with cocktail of vancomycin and bacitracin (Fig. 4a). The genera of hamsters before Abx treatment were distinctly separated from those after Abx treatment based on PCA and PCoA analysis (Fig. 4b, c).

Additionally, there was no significant difference in microbiota at PC1 levels between pea and pork groups, neither before (Pea_Abx_0d vs. Pork_Abx_0d) nor after (Pea_Abx_30d vs. Pork_Abx_30d) feeding. The PC1 levels, accounting for 30.70\% and $75.64 \%$ in PCA and PCoA analysis, respectively, apparently identified that Abx treatment abolished the changes in the gut microbial community generated by diverse proteins (Fig. 4c). Similar clusters were found in the Hierarchical clustering tree at OTU level indicating all samples can be divided into two subgroups containing Abx- and none Abx- (Fig. 4d). No statistical difference was detected in Shannon, Simpson and Chao indices (Fig. 4e-g), but a significant difference was observed in Ace index between pea and pork groups prior to the Abx treatment (Fig. 4h). At the end of experiment, huge declines were observed in both richness and evenness of microbiota, especially the decrease in Ace from 636.77 to 75.52 of Pea_Abx group, and from 648.31 to 68.5 of Prok_Abx group. The same decrease in Chao index was observed in Pea_Abx and Prok_Abx groups from 594.02 to 58.78; 610.66 to 51.92, respectively. As anticipated, the relative abundance of Firmicutes and Bacteroidetes at phylum level were significantly decreased in the presence of continuous Abx administration based on the decreased alpha diversity (Fig. 4i). After the treatment with Abx, Proteobacteria accounting for more than $50 \%$ of total OTU turned to be the dominant bacterial at the phylum level. There were considerable diminutions of specific genera, such as Firmicutes and Bacteroidetes, affected by pea and pork protein supplement as elucidated above (Fig. 2d). Particularly, the difference in abundance of microbiota between pea and pork group disappeared in the presence of Abx, apart from Lactobacillaceae and Bacteroidaceae which accounted for limited in the discrepant bacteria (Fig. 4i-j). These results illustrated that Abx treatment largely eliminated the difference in gut microbiota composition.

Concomitantly, the Abx treatment resulted in an increase in the contents of serum TC, LDL-C and HDL-C of hamsters compared to the hamsters without Abx treatment after 30-day feeding with pea or pork proteins, but there was no significant difference in cholesterol levels between Pea_Abx and Pork_Abx groups (Table 1). It indicated that the inhibitory effects of pea and pork proteins on cholesterol were disappeared with the elimination of gut microbiota induced by Abx. The lipid profiles were significantly different in hamsters treated with Abx from those before treatment. The liver FC, TG and CYP7A1 concentrations were not significantly different between pea and pork groups, but liver TC, CE, HMG-CoA reductase, LDLR, and LPL levels of pea group were significantly lower than those of pork group (Fig. 4k). Notably, the significance of decreases in liver TC induced by pea and pork 
proteins was not such apparent in hamster with Abx treatment $(P<0.05)$ compared to that without Abx treatment $(P<0.001$, Fig. 1f). It implied that the effects of pea and pork protein supplementation on modulating serum cholesterol level were abolished in the absence of gut microbiota in hamster model.

Table 1

Serum lipid contents of hamsters treated with $\mathrm{Abx}(\mathrm{mmol} / \mathrm{L})^{1}$

\begin{tabular}{|c|c|c|c|}
\hline & & Od & $30 d$ \\
\hline \multirow[t]{4}{*}{$\mathrm{TC}$} & Pea_Abx & $3.78 \pm 0.05 a$ & $5.71 \pm 0.07 a$ \\
\hline & Pea & $3.77 \pm 0.15 a$ & $3.47 \pm 0.06 c$ \\
\hline & Pork_Abx & $3.77 \pm 0.04 a$ & $5.82 \pm 0.08 a$ \\
\hline & Pork & $3.77 \pm 0.16 a$ & $3.94 \pm 0.05 b$ \\
\hline \multirow[t]{4}{*}{ LDL-C } & Pea_Abx & $1.20 \pm 0.02 a$ & $2.06 \pm 0.05 a$ \\
\hline & Pea & $1.20 \pm 0.02 a$ & $1.04 \pm 0.03 c$ \\
\hline & Pork_Abx & $1.20 \pm 0.03 a$ & $2.13 \pm 0.14 a$ \\
\hline & Pork & $1.23 \pm 0.02 a$ & $1.26 \pm 0.03 b$ \\
\hline \multirow[t]{4}{*}{ HDL-C } & Pea_Abx & $1.89 \pm 0.02 a$ & $3.38 \pm 0.11 a$ \\
\hline & Pea & $1.82 \pm 0.03 a$ & $1.94 \pm 0.04 b$ \\
\hline & Pork_Abx & $1.89 \pm 0.02 a$ & $3.27 \pm 0.09 a$ \\
\hline & Pork & $1.91 \pm 0.03 a$ & $1.79 \pm 0.02 c$ \\
\hline \multirow[t]{4}{*}{$\mathrm{TG}$} & Pea_Abx & $3.25 \pm 0.13 a$ & $1.22 \pm 0.09 a$ \\
\hline & Pea & $2.93 \pm 0.09 a$ & $1.68 \pm 0.03 b$ \\
\hline & Pork_Abx & $3.25 \pm 0.02 a$ & $1.18 \pm 0.1 a$ \\
\hline & Pork & $2.87 \pm 0.09 a$ & $2.04 \pm 0.03 c$ \\
\hline
\end{tabular}

${ }^{1}$ Means \pm standard errors (SE) were determined for 10 (Pea_Abx and Pork_Abx) and 20 (Pea and pork) hamsters per group. Different superscript letters indicate significant differences at $P<0.05$.

\section{Cross-over intervention of pea and pork proteins reversed cholesterol levels}

Hamsters fed with pea and pork protein in the first month were further respectively divided into 2 groups for the cross-over intervention (Fig. 5a). The corresponded cross-over effects of Pea_Pork and Pork_Pea on the levels of serum TC, LDL-C and TG were observed clearly (Fig. 5b-e). The serum TC and LDL-C levels significantly decreased in pea group while increased in pork group during the first month compare to the original levels before intervention (Fig. 5b, c). Pea protein exhibited obviously higher serum HDL-C and lower TG contents than pork protein (Fig. 5d, e). However, these changes were reversed by the subsequent crossover intervention of proteins after 60 days of feeding. Pork protein intervention led to the highest levels of serum TC and LDL-C which reached to the same levels of Pea_Pork group, but not Pork_Pea. The similar reversed trend were found in pea group which showed the lowest levels of serum TC and LDL-C with the comparable levels of Pork_Pea group, as well as the reversed changes in HDL-C and TG. Moreover, the most body weight gain was presented in Pork_Pea, much higher than the other groups (Table S4). Pea protein intake significantly reduced liver TC and FC contents in contrast to pork protein, and no significant difference was found between Pea_Pork and Pork_Pea in the contents of TC, FC and TG (Fig. 5f). HMG-CoA reductase and LDLR contents were significantly enhanced in pea group compared to pork. Notably, there was no significant difference in the contents of HMG-CoA reductase, CYP7A1, LDLR and LPL between Pea_Pork and Pork_Pea groups (Fig. 5g). These findings confirmed the capacity of pea protein to lower cholesterol, which was strong enough to reverse the side effects of pork protein by dietary shifts. 


\section{Cross-over intervention of pea and pork proteins reshaped gut microbiota}

The alterations of dietary protein from pea to pork and pork to pea both greatly changed hamsters' gut microbiota composition. Pork protein reduced microbiota diversity and evenness significantly based on its lower Shannon, Chao and Ace values and higher Simpson value than pea protein at the first period of 30 days (Fig. S4a-d). The same trend can be observed at the cross-over period when the microbial diversity and evenness of Pork_Pea group were elevated. Notably, no significant difference was found between Pea_Pork and Pork_Pea of which microbial richness and evenness were lower than pea but higher than pork. PCA and PCoA plots for the beta diversity disclosed the significant distinction of the microbial composition structures between pea and pork groups after the first feeding period, while the individual meat groups displayed similar gut microbial structures as reflected by the close clustering within each group (Fig. S4e, f). It's worthy to notice that the farthest distance from pea to pork was just equal to that from Pork_Pea to pork and from Pea_Pork to pea as shown in PCA and PCoA plots (Fig. S4g, h). According to the cluster tree, the two dietary exchange groups came together into one which subsequently joined in pea group for 60 days, and finally formed one clusters with Pork_30d, Pork_60d, and Pea_30d (Fig. S4i). There was a favorable similarity within each group which was consistent with the results of PCA and PCoA.

The dominant phylum were Firmicutes $(64 \% \pm 8 \%)$ and Bacteroidetes $(30 \% \pm 8 \%)$ of all groups identical to the previous results (Fig. 6a). There was a significant difference in the proportions of Firmicutes and Bacteroidetes between pea and pork groups after the first 30 days of feeding. This discrepancy was enhanced at the followed cross-over feeding period, with a significant increase in Bacteroidetes of pea group while decrease of pork. Pea_60d showed no significant difference in the relative abundance of Firmicutes and Bacteroidetes from Pea_Pork_60d or Pork_Pea_60d. Fecal microbiota of hamsters feeding with pork protein was characterized by high norank_f_Eubacteriaceae at the first month, which were almost depleted as converting to pea protein at the second month (Fig. 6b). Pea protein replacing pork also decreased the relative abundance of norank_Erysipelotrichaceae, while increased norank_f_Muribaculaceae and Lachnospiraceae_NK4A136_group. Conversely, norank_f_Eubacteriaceae were greatly declined and Ruminococcus were elevated while norank_f_Muribaculaceae and Lachnospiraceae_NK4A136_group were reduced by substitution pea protein with pork. Two-group comparisons were used to select the different microbiota between pea and pork group at the first feeding period (Fig. 6c). Consumption of pork protein significantly enhanced the relative abundance of Eubacteriaceae while decreased Muribaculaceae, Erysipelotrichaceae, and Ruminococcus compared to pea protein at family level. LEfSe analysis illustrated the important roles of Clostridia mainly contributing to the enrichment of Firmicutes in pork group while Muribaculaceae contributing to the enrichment of Bacteroidetes in pea group accroding to the LDA values (Fig. 6d). Comparisons among these four groups showed significant difference that total proportions of Erysipelotrichaceae and Eubacteriaceae (32.58\%) were higher in Pork group, while the total proportions of Muribaculaceae and Ruminococcaceae were higher in pea, Pea_Pork and Pork_Pea groups, accounting for $56.49 \%, 55.02 \%$ and $58.07 \%$, respectively (Fig. 6e). Likewise, the vital microbiota contributing to the discrepancy relevant to diverse proteins was selected by LEfSe analysis (Fig. 6f). Firmicutes, Bacteroidaceae, Patescibacteria, and Anaeraplasmataceae were respectively enriched in pork, Pork_Pea, Pea_Pork, and pea group. Additionally, the Erysipelotrichia including Erysipelotrichaceae family were of great importance in pork group, while the Muribaculaceae family consisted of norank_Muribaculaceae were enriched in pea group.

The typical diet-dependent microbiotas were identified by two-group comparisons (Fig. 7). Ruminococcaceae, Muribaculaceae, and Lachnospiraceae were identified as the most primary bacteria among all groups, followed by Erysipelotrichaceae. The comparison between pea and pork showed that the most different microbiota with great abundance were Muribaculaceae, followed by Ruminococcaceae, both which were increased in the presence of pea protein, while pork group was characterized by Erysipelotrichaceae and Eubacteriaceae which were barely found in the pea group (Fig. 7a). In Pea_Pork and Pork_Pea groups with the same amount of pea and pork protein intake, there were significant differences in Saccharimonadaceae and Eubacteriaceae accounting for less than $5 \%$ of relative abundance (Fig. 7b). The ability of pea and pork proteins to reshape gut microbiota was next examined by the comparison of cross-over intervention. The significant decreases in proportions of Lachnospiraceae, Erysipelotrichaceae, Rikenellaceae and Eubacteriaceae were observed after changing dietary protein from pea to pork, as well as increases in relative abundance of Ruminococcaceae, Muribaculaceae and Bacteroidaceae (Fig. 7c). Substitution of pork protein with pea significantly increased the relative abundance of Ruminococcaceae, Muribaculaceae and Bacteroidaceae, and almost diminished Erysipelotrichaceae and Eubacteriaceae (Fig. 7d). The gut microbiota composition was similar between pork and Pea_Pork groups; pea and Pork_Pea groups (Fig. 7e, f). These results suggested that dietary exchange of diverse proteins definitely reversed the gut microbiota. 
SCFAs as the key metabolites generated by gut microbiota were beneficial for the lipid homeostasis and cholesterol metabolism and thus were detected, as well as its further indirect effects on cecal metabolites. Pea group showed the highest content of total SCFAs with the primary constituents of acetate, propionate and butyrate, while isobutyrate, isovalerate, and isohexanoate as branched chain fatty acids were not statistically different among the four groups (Table 2). Heat map on basis of metabolite levels showed the highest similarity to the abundance and composition between Pea_Pork and pork, while the lowest correlation between pea and Pea_Pork, suggesting that substitution of pea protein with pork led to the most apparent changes in cecal metabolites (Fig. 8a). Likewise, cross-over diet profoundly altered the cecal metabolites, resulting in the distinct separations that Pea_Pork were close to pork, and Pork_Pea were close to pea in the PCA plot. It implied that the differences caused by dietary exchange of protein were similar to the whole protein itself (Fig. 8b). Among 846 metabolites detection, 497 metabolites were identified with significant different expression quantities by pairwise comparisons with the OPLS-DA models (Fig. 8c, Fig. S5). Based on the databases of HMDB and KEGG, the identified metabolites were classified as lipids and lipid-like molecules; organic acids and derivatives; organoheterocyclic and organic oxygen compounds. Among the 7 categories in KEGG metabolic pathway, most abundant metabolites (228 kinds of metabolite) were annotated in Metabolism type, with 51 kinds of metabolites in lipid metabolism and 40 in amino acid metabolism, followed by Organismal Systems (72 kinds of metabolite) and Human Disease (41 kinds of metabolite) (Fig. 8d, e).

Table 2

Cecal SCFA contents of hamsters with cross-over intervention of pea and pork $(\mu \mathrm{g} / \mathrm{mg})^{1}$

\begin{tabular}{|c|c|c|c|c|c|c|c|c|c|}
\hline & Acetate & Propionate & Isobutyrate & Butyrate & Isovalerate & Valerate & Isohexanoate & Hexanoate & $\begin{array}{l}\text { Total } \\
\text { SCFA }\end{array}$ \\
\hline Pea & $\begin{array}{l}0.6 \pm \\
0.03 a\end{array}$ & $\begin{array}{l}0.25 \pm \\
0.01 a\end{array}$ & $\begin{array}{l}0.06 \pm \\
0.00 \mathrm{a}\end{array}$ & $\begin{array}{l}0.46 \pm \\
0.02 a\end{array}$ & $\begin{array}{l}0.06 \pm \\
0.01 a\end{array}$ & $\begin{array}{l}0.1 \pm \\
0.01 a\end{array}$ & $0.01 \pm 0.00 \mathrm{a}$ & $\begin{array}{l}0.01 \pm \\
0.00 \mathrm{a}\end{array}$ & $\begin{array}{l}1.57 \\
\pm \\
0.03 a\end{array}$ \\
\hline Pea_Pork & $\begin{array}{l}0.43 \pm \\
0.01 \mathrm{~b}\end{array}$ & $\begin{array}{l}0.20 \pm \\
0.01 \mathrm{~b}\end{array}$ & $\begin{array}{l}0.06 \pm \\
0.00 \mathrm{ab}\end{array}$ & $\begin{array}{l}0.25 \pm \\
0.01 \mathrm{c}\end{array}$ & $\begin{array}{l}0.04 \pm \\
0.00 \mathrm{~b}\end{array}$ & $\begin{array}{l}0.07 \pm \\
0.00 \mathrm{ab}\end{array}$ & $0.01 \pm 0.00 \mathrm{~b}$ & $\begin{array}{l}0.01 \pm \\
0.00 \mathrm{a}\end{array}$ & $\begin{array}{l}1.08 \\
\pm \\
0.02 \mathrm{c}\end{array}$ \\
\hline Pork_Pea & $\begin{array}{l}0.46 \pm \\
0.01 \mathrm{~b}\end{array}$ & $\begin{array}{l}0.16 \pm \\
0.01 \mathrm{c}\end{array}$ & $\begin{array}{l}0.05 \pm \\
0.00 \mathrm{~b}\end{array}$ & $\begin{array}{l}0.25 \pm \\
0.01 \mathrm{c}\end{array}$ & $\begin{array}{l}0.03 \pm \\
0.00 \mathrm{bc}\end{array}$ & $\begin{array}{l}0.06 \pm \\
0.00 c\end{array}$ & $0.01 \pm 0.00 \mathrm{ab}$ & $\begin{array}{l}0.01 \pm \\
0.00 a\end{array}$ & $\begin{array}{l}1.04 \\
\pm \\
0.02 \mathrm{c}\end{array}$ \\
\hline Pork & $\begin{array}{l}0.58 \pm \\
0.03 a\end{array}$ & $\begin{array}{l}0.19 \pm \\
0.01 \mathrm{~b}\end{array}$ & $\begin{array}{l}0.05 \pm \\
0.00 \mathrm{~b}\end{array}$ & $\begin{array}{l}0.37 \pm \\
0.01 \mathrm{~b}\end{array}$ & $\begin{array}{l}0.03 \pm \\
0.00 c\end{array}$ & $\begin{array}{l}0.07 \pm \\
0.00 \mathrm{~b}\end{array}$ & $0.01 \pm 0.00 a$ & $\begin{array}{l}0.02 \pm \\
0.00 \mathrm{~b}\end{array}$ & $\begin{array}{l}1.34 \\
\pm \\
0.04 \mathrm{~b}\end{array}$ \\
\hline
\end{tabular}

Pea and pork protein intervention significantly altered fecal metabolites of hamsters with 138 up-regulated and 126 down-regulated metabolites, some of which exhibited differences between pork and pea groups (Fig. 9). Firstly, comparison between pea and pork groups showed that pea protein consumption resulted in significant decreases in oxypinnatanine and glutamylproline concentrations, and increases in 13,14-Dihydro PGF-1a and 4,6-Icosanedione (Fig. 9a, b). Among the top 30 metabolites with high variable importance in projection (VIP) scores, glutamylproline, oxypinnatanine and glycylprolylhydroxyproline belonging to oligopeptide were decreased much more by pea protein. Moreover, the expression of N2-Succinyl-L-ornithine, N-Succinyl-L,L-2,6diaminopimelate and anserine involved in animo acids metabolism were also lower in pea group. Conversely, the sterol lipids compounds like 1a,25-dihydroxy-11alpha-[(1R)-oxiranyl]vitaminD3 and 22-Dehydroclerosterol were enhanced by pea protein (Fig. 9c). Secondly, comparison between the cross-over diet groups and pea or pork groups revealed that 102 up-regulated and 130 down-regulated metabolites in Pea_Pork group compared to pea group (Fig. 9d-f), and 104 up-regulated and 141 down-regulated ones in Pork_Pea group compared to pork group (Fig. 9g-i). It's worthy to notice that the discrepant features were similar between Pea_Pork vs. pea, Pork_Pea vs. pork, and pork vs. pea, especially the relative expression of oxypinnatanine, glutamylproline, 9,10DiHODE, N2-Succinyl-L-ornithine and anserine with high fold change values. Similarly, these metabolites in the volcano plot displayed high VIP scores (Fig. 9d-i). In terms of specific metabolic features, dietary exchange led to the direct change of the 
metabolites away from those in the original diet pattern. The expression of metabolites neither significantly differed between pea and Pork_Pea, nor pork and Pea_Pork. Thirdly, the most effective metabolites with the highest VIP scores were analyzed as show in Fig. 10a-c. The metabolites derived from amino acid metabolism showed that L-arginine was up regulated in pea and Pork_Pea compared to pork group, but N2-Succinyl-L-ornithine, anserine, hercynine, oxypinnatanine and glutamylproline were down regulated (Fig. 10a1-a6). The bile acid metabolites involved in cholesterol metabolism showed that glycocholate was down regulated in pea group, while taurine, 27-Hydroxycholesterol and 3ß,7a-Dihudroxy-5-cholestenoate were up regulated (Fig. 10b1-b4). Other metabolites involved in lipid metabolism showed the increases in sphinganine and 17-Hydroxylinolenic acid in the presence of pea protein, but decrease in 9,10-DiHODE. 12-HETE-GABA belonging to fatty amides was decreased in pea and Pork_Pea groups, while another fatty amide of 1a,25-dihydroxy-11alpha-[(1R)-oxiranyl]vitaminD3 was increased (Fig. 10c1-c5).

The functions of these altered metabolites were identified by KEGG pathway analysis. The significant different pathways common in the pairwise comparisons (pork vs. pea; Pea_Pork vs. pea; pork vs. Pork_Pea) were mainly involved with the amino acid metabolism, including arginine and proline metabolism, D-Arginine and D-ornithine metabolism, and histidine metabolism (Fig. 10d-f). The primary bile acid biosynthesis as one of the most important pathways also contributed to the differences of Pea_Pork from pea. Accordingly, the pathways of amino acid metabolites converted from protein and the pathways of bile acid metabolites converted from cholesterol were identified as shown in Fig. $10 \mathrm{~g}$ and Fig. 10h, respectively. Spearman correlation analysis disclosed the relationship of cecal metabolites with gut microbiota or serum lipid profiles (Fig. 10i, j). The gut microbiotas were divided into three clusters, including left, middle and right clusters, and the cecal metabolites were divided into two clusters, including up and down clusters (Fig. 10i). Metabolites in the up cluster exhibited significantly negative correlation with the microbiotas in the left cluster, while the metabolites in the down cluster displayed positive correlation with these microbiotas in which Erysipelotrichaceae was identified the "harmful" bacteria. On the contrary, the microbiotas in the middle cluster identified as the "beneficial" bacteria, such as Muribaculaceae and Lactobacillus, were positively correlated with metabolites in the up cluster but negatively correlated with metabolites in the down cluster. Correspondingly, metabolites in the up cluster were negatively correlated with serum HDL-C, while positively correlated with serum LDL-C and TC (Fig. 10j). The negative correlation of serum HDL-C was the tightest with N-Succinyl-L,L-2,6-diaminopimelate $(\mathrm{R}=-0.89 . P<0.001)$, followed by feruloyl-agmatine $(\mathrm{R}=-0.79$. $P<$ 0.001). The closest correlation was positively observed between N-Succinyl-L,L-2,6-diaminopimelate and LDL-C $(\mathrm{R}=0.74$. $P<$ $0.001)$, as well as feruloyl-agmatine and TC $(R=0.65 . P<0.001)$. In addition, the closest correlation between microbiotas and metabolites were presented by Eubacteriaceae, followed by Muribaculaceae.

\section{Discussion}

Our present study reveled that the effects of proteins from diverse sources on cholesterol metabolism were closely dependent on gut microbiota susceptible to diet. It was consistent with the previous reports that consumption of plant-based and animal-based diet could alter microbial community structure differently $[19,27,28]$. Moreover, gut microbiota was indeed associated with various circulating metabolites like amino acid and lipid [29-31]. It has been identified Bacteroides were related to metabolism of aromatic and branched chain amino acid in a cohort of obese individuals [32]. Our study initially demonstrated that the different effects of diverse protein on cholesterol regulation were resulted from the alteration of gut microbiota which was confirmed by the Abx treatment experiment. Furthermore, the cross-over proteins of pea and pork could reverse gut microbiota composition and cecal metabolites so that affecting serum cholesterol level.

The plant- and meat- based proteins elicited variation of cholesterol levels in multiple ways which have aroused great attention from researchers. In the early decades, the mechanism of cholesterol-lowering by proteins mainly focused on the hepatic enzymes related to lipid metabolism, such as HMG-CoA reductase and CYP7A1 [33-36]. It attributed the differences in regulation of serum cholesterol or TC levels among different proteins to the ability of plant proteins to inhibit HMG-CoA reductase and stimulate CYP7A1 in the liver of hamster, but not red meat proteins. That was also supported by the present study. In recent years, the composition and structure of gut microbiota have been well evidenced to be associated with cholesterol homeostasis [37]. Muribaculaceae, cultured and identified from the bacterial family S24-7, were negatively correlated with serum TC and TG, and were strongly associated with SCFAs secretion [38-40]. The enhanced diversity of gut microbiota is crucial for protection of intestinal function and reduced diversity was always accompanied by obesity and a diet with much sugar and fat [41-43]. Our study elucidated that plant protein induced higher bacterial diversity and Muribaculaceae proportion than meat protein. For example, pea group with higher diversity than pork group was rich in relative abundance of Eubacteriaceae, Erysipelotrichaceae, Muribaculaceae,

Page $12 / 30$ 
Ruminococcaceae and Lachnospirace. Muribaculaceae played the especially key role in contributing to the significant difference among all the groups, which were enriched in oat and pea groups with higher contents of SCFAs than red meat groups. Conversely, the red meat groups showed significantly higher proportions of Eubacteriaceae and Erysipelotrichaceae than plant groups. Accumulating abundance of Erysipelotrichaceae in gut lumina of the mammal model of colon cancer was associated with inflammatory bowel diseases, and its reduction was observed in hamster model of hypercholesterolemia [37, 44]. The present study demonstrated the key role of "beneficial" microbe Muribaculaceae in responsible for the cholesterol regulation of plant proteins, especially pea and oat proteins, as well as the role of "harmful" microbe Erysipelotrichaceae for the cholesterol regulation of meat proteins.

The discrepancy in the gut microbial community between plant and red meat groups was profoundly due to the different AA compositions of different proteins. Glu, Cys, Val, and Ser with the most proportion in plant proteins were positively correlated with the abundance of norank_f_Muribaculaceae, Eubacterium_ruminantium_group and Lactobacillus, while Thr, His and Lys with less proportion than the four above were negatively correlated with these genera. Norank_f_Muribaculaceae,

Eubacterium_ruminantium_group and Lactobacillus showed negative association with serum and liver cholesterol concentrations while positive association with SCFAs, which confirmed the beneficial effects of these bacteria on cholesterol regulation. The further correlation analysis also supported this finding that the increase in abundance of these three bacteria corresponded the lowering levels of TC, LDL-C, and TG, and thus suggested the key role of amino acid compositions in lowering lipid and cholesterol levels. Essential amino acids as a source of nitrogen and sulfur are irreplaceable for their enormous physiological importance [45]. Glutamine supplementation can mitigate the waist circumference and blood lipopolysaccharide in obese individuals by modulating gut microbiota [46]. Val is an essential substrate involved in protein metabolism because it contributes to gut bacterial improvement of piglets [47]. In the present study, the proportions of Glu and Val are higher in plant groups than those in meat groups, and corresponded with three main genera enrichment which were not characterized or cultured until now [18]. Therefore, diverse proteins have effects on the cholesterol metabolism not only by the absorption as amino acid or peptides in small intestine, but also probably in the form of metabolites from amino acid in large intestine, which was illustrated by analyzing cecal metabolites in the next cross-over intervention of pea and pork proteins.

The mechanism of direct association between gut microbiota and cholesterol-regulation of different proteins were next clarified by bacterial catabolism of protein and its hydrolysate in hamster. The decisive roles of gut microbiota were explored by hamsters with Abx treatment. The near complete elimination of specific species and abolished discrepancies in gut microbiota were caused by continuous Abx treatment in response to diverse proteins interventions. These results agreed with the previous findings of Scott et al. who observed that Abx treatment eliminated the effects of tryptophan generated by gut microbiota catabolism [48]. The Abx treatment was also reported to deplete most microbiota and abolish the beneficial effects of proanthocyanidin from grape seed on high-fat diet fed mice [49]. The difference in two predominant gut microbiotas, Firmicutes and Bacteroidetes, between pea and pork groups were disappeared, together with no significant difference in the serum and liver lipid profiles in the presence of Abx administration. As the abundance of Firmicutes and Bacteroidetes diminished largely, the Proteobacteria elevated correspondly [26]. Proteobacteria, as one of the most abundant bacteria, have the potential to drive chronic colitis in mice involved in cholesterol metabolism [50]. In the present study, the abnormal higher TC and LDL-C were observed in the hamster treated with Abx, together with the elimination of differences in cholesterol levels between pea and pork, supporting the decisive role of gut microbiota in cholesterol regulation.

Given gut microbiota were so important for the lowering-cholesterol effects of diverse proteins as mentioned above, whether immediate change in diet could reshape the gut microbiota was necessary to be investigated. The cross-over interventions with pea and pork protein confirmed this necessary reconfiguration in our subsequent study. Accordingly, the dynamic reversement of gut microbial community was observed clearly when the diet converted from pea to pork or pork to pea. Pea_Pork group showed the similar changes in gut microbiota structures and lipid profiles to pork gourp, and Pork_Pea group was similar to pea group based on the results of cluster analysis. The close correlation of dynamic reversement by diet with gut microbiota and further lipid profiles reconfirmed that diets alteration of appropriate protein could have beneficial effect on lipid metabolism by modulating the gut microbiota structure. Firmicutes and Bacteroidetes are the two dominant phyla collectively accounting for $94 \%$ of total taxa at the end of experiment which is in line with the findings on gut microbiota at the phylum level mention above [51]. The cross-over intervention from pork to pea protein significantly increased the proportions of Muribaculaceae, Ruminococcaceae and Lachnospirace with correspondingly decreased Eubacteriaceae and Erysipelotrichaceae, which was coincident with the effects of

Page $13 / 30$ 
pea protein. The replacement of pea protein with pork exhibited no profound changes in the beneficial gut microbiota such as Muribaculaceae and Ruminococcaceae, which could be attributed to the strong resilience of gut microbiota induced by pea protein. The gut microbiota has been reported to restore the equilibrium of gut ecosystem to the external challenges that is resilience [52]. In contrast, the replacement of pork protein with pea significantly reserved the gut microbiota compositions according to the diminution in probiotics like Muribaculaceae, Ruminococcaceae [53], and accumulation in the potential pathogenic bacteria Erysipelotrichaceae [54]. These evidences of "beneficial" microbe Muribaculaceae mainly responsible for pea protein effects and "harmful" microbe Erysipelotrichaceae for pork protein was consistent with the above results of gut microbiota compositions. The contrary alterations of gut microbiota found in the diet exchange possibly resulted from the alterations of multiple metabolites induced by pea protein supplemented in complex metabolic pathways [55], which was illustrated next.

The metabolites in the caecum were produced from the food residue utilized by gut microbiota. The significant changes in cecal metabolites in response to diverse proteins were mainly classified as the amino acid derivatives derived from protein metabolism, bile acids biosynthesis converted from cholesterol metabolism, SCFAs secretion produced from gut microbiota or lipid and lipid-like molecules involved in lipid metabolism. Firstly, the differences in amino acids metabolites between pea and pork protein were mainly attributed to the arginine metabolism pathway, and histidine metabolism pathway. The effect of pea protein was not only related to the arginine but also closely involved with its luminal metabolites, based on the results of down-regulated N2-Succinyl-Lornithine and feruloyl-agmatine, but up-regulated creatine. L-arginine supplementation has been reported to enhance whole-body insulin sensitivity and reduce plasma lipid and cholesterol levels $[56,57]$. In addition to the direct benefits, accumulating gut luminal arginine was also closely associated with the circulating amino acid metabolism by interacting with gut microbiotas to product relevant metabolites $[32,58]$. However, the correlation between L-arginine metabolites and gut microbiota relevant to cholesterol metabolism was not as clear as the function of L-arginine itself based on the plasma absorption. Our study discovered that the metabolism of arginine/creatine pathway was beneficial for the lowering-chelesterol, rather than arginine/N2-Succinyl-Lornithine or / feruloyl-agmatine pathways. Although the content of arginine in pork protein was higher than that in pea protein, the gut luminal arginine of hamster feeding with pea protein was much more than that of pork protein, resulting in that more arginine promoted more growth of "beneficial" bacteria, such as Muribaculaceae and Lactobacillus, and their interaction produced more "beneficial" metabolites, such as creatine which beneficial for the cholesterol regulation. Conversely, less gut luminal arginine of hamster feeding with pork protein led to the more growth of "harmful" bacteria, such as Erysipelotrichaceae, and further produced more "harmful" metabolites, such as N2-Succinyl-L-ornithine and feruloyl-agmatine which have negative effect on the cholesterol regulation of pork protein. As the same as histidine metabolism, the pathways of anserine or hercynine were inhibited in the presence of pea protein so as to promote the probiotics growth and suppress cholesterol levels. Furthermore, oxypinnatanine and glutamylproline as unknown metabolites of amino acid might identify to be unfavorable for lowering-cholesterol regulation.

Secondly, primary bile acid biosynthesis involved in cholesterol metabolism was also affected by the dietary exchange from pork to pea protein. A key intermediate product from cholesterol to cholate, glycocholate, was observed to positively correlate with TC, LDLC while negatively correlate with HDL-C, which was also evidenced by Guo et al. [59] who reported the potential roles of gut microbiota and bile acids in antidiabetic treatment. Primary bile acids are synthesized in the liver and discharged into intestine where the conversion of cholesterol into bile acids occurs, and then subject to extensive metabolism by gut microbes, namely deconjugation of glycine or taurine, and biotransformation of the unconjugated primary bile acids to secondary bile acids [60, 61]. This complex transformation of primary and secondary bile acids is crucial for their biological function contributing to absorption of dietary lipids [62]. In the present study, pork protein resulted in the significant inhibition of acidic pathway and the stimulation of neutral pathway through which cholesterol converted to cholate by glycocholate pathway. However, pea protein promoted the production of cholate by taurine pathway, and its effect on chenodeoxycholate synthesis by 27 -Hydroxycholesterol/3 $\beta, 7 a-$ Dihudroxy-5-cholestenoate pathway was more significant, indicating the close relationship between bile acid biosynthesis and the changes in gut microbiota and cholesterol regulation.

Thirdly, the differences between pea and pork protein were also associated with the lipid like metabolism, such as sphingomyelin/ceramide pathway activation plays a crucial role in oxidized LDLs induced atherosclerotic lesions [63]; 17Hydroxylinolenic acid or 9,10-DiHODE as linolenlic metabolites indeed regulate the lipid profiles by inhibition on HMG-CoA activity [64]; tow fatty amides, 12-HETE-GABA and 1a,25-dihydroxy-11alpha-[(1R)-oxiranyl]vitaminD3, also contribute to the diverse effects of protein. Consistently, as productions of gut microbiota, the SCFAs contents in the caecum, including acetate, propionate and butyrate, were significantly increased by pea protein intervention together with the increase in relative abundance of

Page $14 / 30$ 
Muribaculaceae, which supported their positive association as reported by previous studies [39, 40,53]. These metabolites alterations resulting from gut microbiota corresponded to the changes in lipid and cholesterol levels induced by the diverse proteins. Gut microbiota can utilize digestion products of food ingredients that diverse proteins produce different nutrients for gut microbiota to generate various metabolites, in turn, to involve in host metabolism [65]. Therefore, the lowering-cholesterol effects of pea protein can be attributed to it promotion of beneficial gut microbiota like Muribaculaceae and Lachnospirace, which resulted in the corresponded regulation of cecal metabolites involved in amino acid, bile acid and lipid metabolism.

\section{Conclusion}

It is the first time to identify the different cholesterol-regulating effects of proteins from diverse sources and correlate these differences with changes in gut mictobita so as to clarify the effective mechanism on cholesterol metabolism. Plant proteins such as pea protein showed significantly lower serum or liver cholesterol levels than meat proteins such as pork protein, which corresponded to the changes in gut microbiota including the increased abundances of Muribaculaceae by pea protein and Erysipelotrichaceae by pork protein. The decisive roles of gut microbiota were confirmed by the findings that no more difference in serum or liver cholesterol was observed in the presence of Abx with the elimination of gut microbiota. The effects of gut microbiota on cholesterol metabolism depended on the dietary pattern which was reversible by shifting protein types. The relevant SCFAs levels in responsible for Muribaculaceae and Erysipelotrichaceae were also distinguished between pea and pork proteins. As the same as the cecal metabolites induced by diverse proteins supplement, cross-over intervention either from pork to pea or from pea to pork exerted led to the reversed changes in arginine, taurine, proline metabolism and sphingomyelin/ceramide pathway. Overall, our study demonstrated that the difference in cholesterol regulation between plant and meat proteins could be attributed to the improvement of amino acid metabolism, bile acid biosynthesis and SCFAs secretion, which depended on gut microbiota, and, in comparison, consumption of pea protein was beneficial for the cholesterol regulation by stimulating the growth of beneficial bacteria of Muribaculaceae.

\section{Abbreviations}

Abx: antibiotic; ACAT: cholesterol acyltransferase; ANOVA: analysis of variance; Apo: apolipoproteins; CE: cholesterol ester; CVD: cardio-cerebrovascular disease; CYP7A1: cholesterol 7a-hydroxylase; FAS: fatty acid synthase; FC: free cholesterol; HDL-C: highdensity lipoprotein cholesterol; HMG-CoA: 3-hydroxy-3-methyl glutaryl coenzyme A; LDA: Linear discriminant analysis; LDL-C: lowdensity lipoprotein cholesterol; LDLR: low density lipoprotein receptor; LEfSe: Linear discriminant analysis coupled with effect size measurements; LPL: lipoprotein lipase; OPLS-DA: orthogonal partial least squares discriminant analysis; OTU: operational taxonomic units; PCA: Principal coordinates analysis; PCoA: and principal coordinate analysis; RDA: Redundancy analysis; SCFAs: short chain fatty acids; TC: total cholesterol; TG: triglyceride; VIP: variable importance in projection.

\section{Declarations}

\section{Ethics approval and consent to participate}

All animal procedures in this study were performed in accordance with the guidelines approved by the Ethical Committee of Experimental Animal Center of the Institute of Medicinal Plant Development, Chinese Academy of Medical Sciences \& Peking Union Medical College.

\section{Consent for publication}

Not applicable

\section{Availability of data and materials}

The biochemical data in this work are available at GitHub (https://github.com/82101182149/Protein_Microbiome_Journal.git). The raw data were deposited to NCBI SRA database and are available for download under BioProject accession code PRJNA689329. The raw data of untargeted metabolome analysis are freely sccessible at GitHub (https://github.com/82101182149/MetabolitesMicrobiome-Journal.git).

Page $15 / 30$ 


\section{Competing interests}

The authors declare no competing interests.

\section{Funding}

This work was supported by China agriculture research system (CARS-08-G19).

This work was supported by Soy industry technology system, China (CARS-02-PS29).

This work was supported by Food nutrition and functional factor utilization team of the Agricultural Science and Technology Innovation Program, CAAS.

\section{Authour's contributions}

LTT and FZW designed and supervised this study. TZX and ACW prepared the samples and conducted the animal experiments. TZX, WYQ and XRZ performed the data analysis. LLW, CL and LYL assisted in statistical analysis of the metadata. LTT and TZX wrote the manuscript. All authors read and approved the final manuscript.

\section{Acknowledgements}

Not applicable.

\section{References}

1. Micha R, Wallace SK, Mozaffarian D. Red and processed meat consumption and risk of incident coronary heart disease, stroke, and diabetes mellitus: a systematic review and meta-analysis. Circulation. 2010;121(21):2271-83.

2. Pan A, Sun Q, Bernstein AM, Schulze MB, Manson JE, Stampfer MJ, Willett WC, Hu FB. Red meat consumption and mortality: results from 2 prospective cohort studies. Arch Intern Med. 2012;172(7):555-63.

3. O'Connor LE, Kim JE, Campbell WW. Total red meat intake of $\geq 0.5$ servings/d does not negatively influence cardiovascular disease risk factors: a systemically searched meta-analysis of randomized controlled trials. Am J Clin Nutr. 2017;105(1):5769.

4. Naghshi S, Sadeghi O, Willett WC, Esmaillzadeh A. Dietary intake of total, animal, and plant proteins and risk of all cause, cardiovascular, and cancer mortality: systematic review and dose-response meta-analysis of prospective cohort studies. BMJ. 2020;370:m2412.

5. Budhathoki S, Sawada N, Iwasaki M, Yamaji T, Goto A, Kotemori A, Ishihara J, Takachi R, Charvat H, Mizoue T, Iso H, Tsugane S. Association of animal and plant protein intake with all-cause and cause-specific mortality in a Japanese Cohort. JAMA Intern Med. 2019;179(11):1509-18.

6. Guasch-Ferré M, Satija A, Blondin SA, Janiszewski M, Emlen E, O'Connor LE, Campbell WW, Hu FB, Willett WC, Stampfer MJ. Meta-analysis of randomized controlled trials of red meat consumption in comparison with various comparison diets on cardiovascular risk factors. Circulation. 2019;139(15):1828-45.

7. Ferdowsian HR, Barnard ND. Effects of plant-based diets on plasma lipids. Am J Cardiol. 2009;104(7):947-56.

8. Yokoyama Y, Levin SM, Barnard ND. Association between plant-based diets and plasma lipids: a systematic review and metaanalysis. Nutr Rev. 2017;75(9):683-98.

9. Morita T, Oh-hashi A, Takei K, Ikai M, Kasaoka S, Kiriyama S. Cholesterol-lowering effects of soybean, potato and rice proteins depend on their low methionine contents in rats fed a cholesterol-free purified diet. J Nutr. 1997;127(3):470-7.

10. Tomotake H, Shimaoka I, Kayashita J, Yokoyama F, Nakajoh M, Kato N. A buckwheat protein product suppresses gallstone formation and plasma cholesterol more strongly than soy protein isolate in hamsters. J Nutr. 2000;130(7):1670-4.

11. Tong LT, Fujimoto Y, Shimizu N, Tsukino M, Akasaka T, Kato Y, Iwamoto W, Shiratake S, Imaizumi K, Sato M. Rice a-globulin decreases serum cholesterol concentrations in rats fed a hypercholesterolemic diet and ameliorates atherosclerotic lesions in apolipoprotein E-deficient mice. Food Chem. 2012;132(1):194-200.

Page $16 / 30$ 
12. Yang L, Han G, Liu QH, Wu Q, He HJ, Cheng CZ, Duan YJ. Rice protein exerts a hypocholesterolemic effect through regulating cholesterol metabolism-related gene expression and enzyme activity in adult rats fed a cholesterol-enriched diet. Int J Food Sci Nutr. 2013;64(7):836-42.

13. Tong LT, Guo L, Zhou X, Qiu J, Liu L, Zhong K, Zhou S. Effects of dietary oat proteins on cholesterol metabolism of hypercholesterolaemic hamsters. J Sci Food Agric. 2016;96(4):1396-401.

14. Rigamonti E, Parolini C, Marchesi M, Diani E, Brambilla S, Sirtori CR, Chiesa G. Hypolipidemic effect of dietary pea proteins: impact on genes regulating hepatic lipid metabolism. Mol Nutr Food Res. 2010;54 Suppl 1:S24-30.

15. ljaz MU, Ahmed MI, Zou X, Hussain M, Zhang M, Zhao F, Xu X, Zhou G, Li C. Beef, casein, and soy proteins differentially affect lipid metabolism, triglycerides accumulation and gut microbiota of high-fat diet-fed C57BL/6J mice. Front Microbiol. 2018;9:2200.

16. Butteiger DN, Hibberd AA, McGraw NJ, Napawan N, Hall-Porter JM, Krul ES. Soy protein compared with milk protein in a western diet increases gut microbial diversity and reduces serum lipids in golden syrian hamsters. J Nutr. 2016;146(4):697705.

17. Eyssen HJ, Parmentier GG, Compernolle FC, De Pauw G, Piessens-Denef M. Biohydrogenation of sterols by Eubacterium ATCC 21,408-Nova species. Eur J Biochem. 1973;36(2):411-21.

18. Kenny DJ, Plichta DR, Shungin D, Koppel N, Hall AB, Fu B, Vasan RS, Shaw SY, Vlamakis H, Balskus EP, Xavier RJ. Cholesterol metabolism by uncultured human gut bacteria influences host cholesterol level. Cell Host Microbe. 2020;28(2):245-57.e6.

19. David LA, Maurice CF, Carmody RN, Gootenberg DB, Button JE, Wolfe BE, Ling AV, Devlin AS, Varma Y, Fischbach MA, Biddinger SB, Dutton RJ, Turnbaugh PJ. Diet rapidly and reproducibly alters the human gut microbiome. Nature. 2014;505(7484):55963.

20. Sonnenburg ED, Smits SA, Tikhonov M, Higginbottom SK, Wingreen NS, Sonnenburg JL. Diet-induced extinctions in the gut microbiota compound over generations. Nature. 2016;529(7585):212-5.

21. Day, L. Proteins from land plants-potential resources for human nutrition and food security. Trends in Food Sci Tech. 2013;32(1):25-42.

22. Dahl WJ, Foster LM, Tyler RT. Review of the health benefits of peas (Pisum sativum L.). Br J Nutr. 2012;108 Suppl 1:S3-10.

23. Abeysekara S, Chilibeck PD, Vatanparast $H$, Zello GA. A pulse-based diet is effective for reducing total and LDL-cholesterol in older adults. Br J Nutr. 2012;108 Suppl 1:S103-10.

24. István, S, Vida V. Global tendencies in pork meat-production, trade and consumption. Applied Studies in Agribusiness and Commerce. 2017;11(3-4):105-11.

25. Ley RE, Hamady M, Lozupone C, Turnbaugh PJ, Ramey RR, Bircher JS, Schlegel ML, Tucker TA, Schrenzel MD, Knight R, Gordon JI. Evolution of mammals and their gut microbes. Science. 2008;320(5883):1647-51.

26. Hwang I, Park YJ, Kim YR, Kim YN, Ka S, Lee HY, Seong JK, Seok YJ, Kim JB. Alteration of gut microbiota by vancomycin and bacitracin improves insulin resistance via glucagon-like peptide 1 in diet-induced obesity. FASEB J. 2015;29(6):2397-411.

27. Devkota S, Wang Y, Musch MW, Leone V, Fehlner-Peach H, Nadimpalli A, Antonopoulos DA, Jabri B, Chang EB. Dietary-fatinduced taurocholic acid promotes pathobiont expansion and colitis in II10-/- mice. Nature. 2012;487(7405):104-8.

28. Raman AS, Gehrig JL, Venkatesh S, Chang HW, Hibberd MC, Subramanian S, Kang G, Bessong PO, Lima AAM, Kosek MN, Petri WA Jr, Rodionov DA, Arzamasov AA, Leyn SA, Osterman AL, Huq S, Mostafa I, Islam M, Mahfuz M, Haque R, Ahmed T, Barratt MJ, Gordon JI. A sparse covarying unit that describes healthy and impaired human gut microbiota development. Science. 2019;365(6449):eaau4735.

29. Vojinovic D, Radjabzadeh D, Kurilshikov A, Amin N, Wijmenga C, Franke L, Ikram MA, Uitterlinden AG, Zhernakova A, Fu J, Kraaij $\mathrm{R}$, van Duijn CM. Relationship between gut microbiota and circulating metabolites in population-based cohorts. Nat Commun. 2019;10(1):5813.

30. Fu J, Bonder MJ, Cenit MC, Tigchelaar EF, Maatman A, Dekens JA, Brandsma E, Marczynska J, Imhann F, Weersma RK, Franke L, Poon TW, Xavier RJ, Gevers D, Hofker MH, Wijmenga C, Zhernakova A. The gut microbiome contributes to a substantial proportion of the variation in blood lipids. Circ Res. 2015;117(9):817-24.

31. Rothschild D, Weissbrod O, Barkan E, Kurilshikov A, Korem T, Zeevi D, Costea PI, Godneva A, Kalka IN, Bar N, Shilo S, Lador D, Vila AV, Zmora N, Pevsner-Fischer M, Israeli D, Kosower N, Malka G, Wolf BC, Avnit-Sagi T, Lotan-Pompan M, Weinberger A,

Page $17 / 30$ 
Halpern Z, Carmi S, Fu J, Wijmenga C, Zhernakova A, Elinav E, Segal E. Environment dominates over host genetics in shaping human gut microbiota. Nature. 2018;555(7695):210-5.

32. Liu R, Hong J, Xu X, Feng Q, Zhang D, Gu Y, Shi J, Zhao S, Liu W, Wang X, Xia H, Liu Z, Cui B, Liang P, Xi L, Jin J, Ying X, Wang X, Zhao X, Li W, Jia H, Lan Z, Li F, Wang R, Sun Y, Yang M, Shen Y, Jie Z, Li J, Chen X, Zhong H, Xie H, Zhang Y, Gu W, Deng X, Shen B, Xu X, Yang H, Xu G, Bi Y, Lai S, Wang J, Qi L, Madsen L, Wang J, Ning G, Kristiansen K, Wang W. Gut microbiome and serum metabolome alterations in obesity and after weight-loss intervention. Nat Med. 2017;23(7):859-68.

33. Sato K, Ohuchi A, Sook SH, Toyomizu M, Akiba Y. Changes in mRNA expression of 3-hydroxy-3-methylglutaryl coenzyme A reductase and cholesterol 7 alpha-hydroxylase in chickens. Biochim Biophys Acta. 2003;1630(2-3):96-102.

34. Li T, Matozel M, Boehme S, Kong B, Nilsson LM, Guo G, Ellis E, Chiang JY. Overexpression of cholesterol 7a-hydroxylase promotes hepatic bile acid synthesis and secretion and maintains cholesterol homeostasis. Hepatology. 2011;53(3):9961006.

35. Zhang H, Bartley GE, Mitchell CR, Zhang H, Yokoyama W. Lower weight gain and hepatic lipid content in hamsters fed high fat diets supplemented with white rice protein, brown rice protein, soy protein, and their hydrolysates. J Agric Food Chem. 2011;59(20):10927-33.

36. Zhang C, Zhang R, Li YM, Liang N, Zhao Y, Zhu H, He Z, Liu J, Hao W, Jiao R, Ma KY, Chen ZY. Cholesterol-lowering activity of tartary buckwheat protein. J Agric Food Chem. 2017;65(9):1900-6.

37. Oliphant $\mathrm{K}$, Allen-Vercoe E. Macronutrient metabolism by the human gut microbiome: major fermentation by-products and their impact on host health. Microbiome. 2019;7:91.

38. Martínez I, Wallace G, Zhang C, Legge R, Benson AK, Carr TP, Moriyama EN, Walter J. Diet-induced metabolic improvements in a hamster model of hypercholesterolemia are strongly linked to alterations of the gut microbiota. Appl Environ Microbiol. 2009;75(12):4175-84.

39. Lagkouvardos I, Lesker TR, Hitch TCA, Gálvez EJC, Smit N, Neuhaus K, Wang J, Baines JF, Abt B, Stecher B, Overmann J, Strowig T, Clavel T. Sequence and cultivation study of Muribaculaceae reveals novel species, host preference, and functional potential of this yet undescribed family. Microbiome. 2019;7:28.

40. Smith BJ, Miller RA, Ericsson AC, Harrison DC, Strong R, Schmidt TM. Changes in the gut microbiome and fermentation products concurrent with enhanced longevity in acarbose-treated mice. BMC Microbiol. 2019;19(1):130.

41. Sommer F, Anderson JM, Bharti R, Raes J, Rosenstiel P. The resilience of the intestinal microbiota influences health and disease. Nat Rev Microbiol. 2017;15(10):630-8.

42. Turnbaugh PJ, Bäckhed F, Fulton L, Gordon JI. Diet-induced obesity is linked to marked but reversible alterations in the mouse distal gut microbiome. Cell Host Microbe. 2008;3(4):213-23.

43. Yatsunenko T, Rey FE, Manary MJ, Trehan I, Dominguez-Bello MG, Contreras M, Magris M, Hidalgo G, Baldassano RN, Anokhin AP, Heath AC, Warner B, Reeder J, Kuczynski J, Caporaso JG, Lozupone CA, Lauber C, Clemente JC, Knights D, Knight R, Gordon JI. Human gut microbiome viewed across age and geography. Nature. 2012;486(7402):222-7.

44. Schaubeck M, Clavel T, Calasan J, Lagkouvardos I, Haange SB, Jehmlich N, Basic M, Dupont A, Hornef M, von Bergen M, Bleich A, Haller D. Dysbiotic gut microbiota causes transmissible Crohn's disease-like ileitis independent of failure in antimicrobial defence. Gut. 2016;65(2):225-37.

45. Wu G. Dietary protein intake and human health. Food Funct. 2016;7(3):1251-65.

46. Abboud KY, Reis SK, Martelli ME, Zordão OP, Tannihão F, de Souza AZZ, Assalin HB, Guadagnini D, Rocha GZ, Saad MJA, Prada PO. Oral glutamine supplementation reduces obesity, pro-inflammatory markers, and improves insulin sensitivity in DIO wistar rats and reduces waist circumference in overweight and obese humans. Nutrients. 2019;11(3):536.

47. Yin J, Ma J, Li Y, Ma X, Chen J, Zhang H, Wu X, Li F, Liu Z, Li T, Yin Y. Branched-chain amino acids, especially of leucine and valine, mediate the protein restricted response in a piglet model. Food Funct. 2020;11(2):1304-11.

48. Scott SA, Fu J, Chang PV. Microbial tryptophan metabolites regulate gut barrier function via the aryl hydrocarbon receptor. Proc Natl Acad Sci U S A. 2020;117(32):19376-87.

49. Liu W, Zhao S, Wang J, Shi J, Sun Y, Wang W, Ning G, Hong J, Liu R. Grape seed proanthocyanidin extract ameliorates inflammation and adiposity by modulating gut microbiota in high-fat diet mice. Mol Nutr Food Res. 2017;61:9. 
50. Carvalho FA, Koren O, Goodrich JK, Johansson ME, Nalbantoglu I, Aitken JD, Su Y, Chassaing B, Walters WA, González A, Clemente JC, Cullender TC, Barnich N, Darfeuille-Michaud A, Vijay-Kumar M, Knight R, Ley RE, Gewirtz AT. Transient inability to manage proteobacteria promotes chronic gut inflammation in TLR5-deficient mice. Cell Host Microbe. 2012;12(2):139-52.

51. Ley RE, Turnbaugh PJ, Klein S, Gordon JI. Microbial ecology: human gut microbes associated with obesity. Nature. 2006;444(7122):1022-3.

52. Fassarella M, Blaak EE, Penders J, Nauta A, Smidt H, Zoetendal EG. Gut microbiome stability and resilience: elucidating the response to perturbations in order to modulate gut health. Gut. 2020;0:1-11.

53. Shang Q, Shan X, Cai C, Hao J, Li G, Yu G. Dietary fucoidan modulates the gut microbiota in mice by increasing the abundance of Lactobacillus and Ruminococcaceae. Food Funct. 2016;7(7):3224-32.

54. Kaakoush NO. Insights into the role of Erysipelotrichaceae in the human host. Front Cell Infect Microbiol. 2015;5:84.

55. Le Chatelier E, Nielsen T, Qin J, Prifti E, Hildebrand F, Falony G, Almeida M, Arumugam M, Batto JM, Kennedy S, Leonard P, Li J, Burgdorf K, Grarup N, Jørgensen T, Brandslund I, Nielsen HB, Juncker AS, Bertalan M, Levenez F, Pons N, Rasmussen S, Sunagawa S, Tap J, Tims S, Zoetendal EG, Brunak S, Clément K, Doré J, Kleerebezem M, Kristiansen K, Renault P, SicheritzPonten T, de Vos WM, Zucker JD, Raes J, Hansen T; MetaHIT consortium, Bork P, Wang J, Ehrlich SD, Pedersen O. Richness of human gut microbiome correlates with metabolic markers. Nature. 2013;500(7464):541-6.

56. Moon J, Kim OY, Jo G, Shin MJ. Alterations in circulating amino acid metabolite ratio associated with arginase activity are potential indicators of metabolic syndrome: the Korean genome and epidemiology study. Nutrients. 2017;9(7):740.

57. Dashtabi A, Mazloom Z, Fararouei M, Hejazi N. Oral L-Arginine administration improves anthropometric and biochemical indices associated with cardiovascular diseases in obese patients: a randomized, single blind placebo controlled clinical trial. Res Cardiovasc Med. 2015;5(1):e29419.

58. Claeyssens S, Lecleire S, Leblond J, Marion R, Hecketsweiler B, Lavoinne A, Ducrotté P, Déchelotte P, Coëffier M. Lack of effect of acute enteral arginine infusion on whole-body and intestinal protein metabolism in humans. Dig Dis Sci. 2007;52(8):182632.

59. Gu Y, Wang X, Li J, Zhang Y, Zhong H, Liu R, Zhang D, Feng Q, Xie X, Hong J, Ren H, Liu W, Ma J, Su Q, Zhang H, Yang J, Wang X, Zhao X, Gu W, Bi Y, Peng Y, Xu X, Xia H, Li F, Xu X, Yang H, Xu G, Madsen L, Kristiansen K, Ning G, Wang W. Analyses of gut microbiota and plasma bile acids enable stratification of patients for antidiabetic treatment. Nat Commun. 2017;8(1):1785.

60. Heinken A, Ravcheev DA, Baldini F, Heirendt L, Fleming RMT, Thiele I. Systematic assessment of secondary bile acid metabolism in gut microbes reveals distinct metabolic capabilities in inflammatory bowel disease. Microbiome. 2019;7(1):75.

61. Wahlström A, Sayin SI, Marschall HU, Bäckhed F. Intestinal crosstalk between bile acids and microbiota and its impact on host metabolism. Cell Metab. 2016;24(1):41-50.

62. Derrien M, van Hylckama Vlieg JE. Fate, activity, and impact of ingested bacteria within the human gut microbiota. Trends Microbiol. 2015;23(6):354-66.

63. Augé N, Maupas-Schwalm F, Elbaz M, Thiers JC, Waysbort A, Itohara S, Krell HW, Salvayre R, Nègre-Salvayre A. Role for matrix metalloproteinase-2 in oxidized low-density lipoprotein-induced activation of the sphingomyelin/ceramide pathway and smooth muscle cell proliferation. Circulation. 2004;110(5):571-8.

64. Schuchardt JP, Schmidt S, Kressel G, Dong H, Willenberg I, Hammock BD, Hahn A, Schebb NH. Comparison of free serum oxylipin concentrations in hyper- vs. normolipidemic men. Prostag Leukotr Ess. 2013;89(1):19-29.

65. Alexander M, Turnbaugh PJ. Deconstructing mechanisms of diet-microbiome-immune interactions. Immunity. 2020;53(2):26476.

\section{Figures}



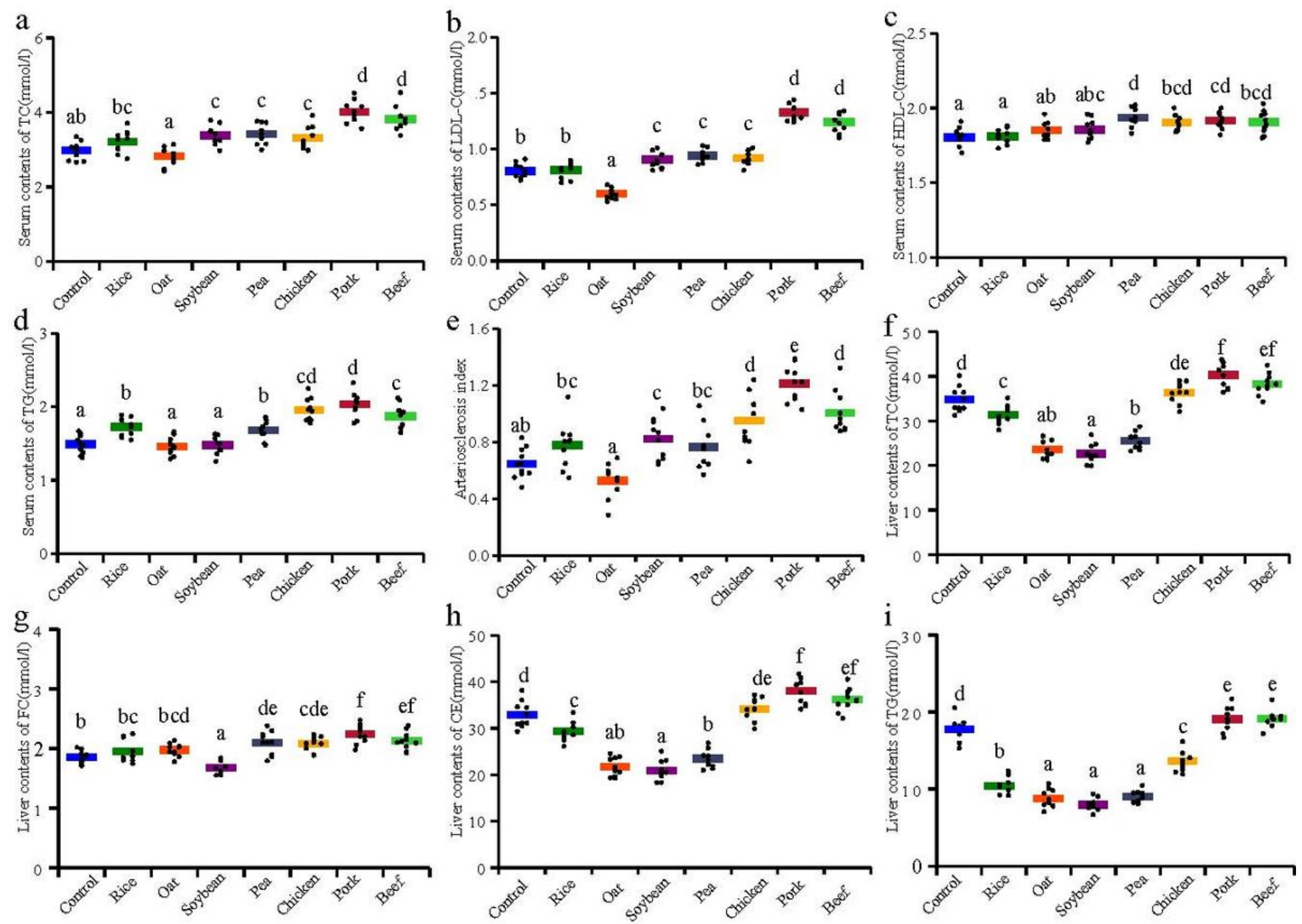

Figure 1

Effects of diverse proteins on serum and liver lipid profiles. a-d Serum levels of TC: total cholesterol (a); LDL-C: low density lipoprotein cholesterol (b); HDL-C: high density lipoprotein cholesterol (c); TG: triglycerides (d); e arteriosclerosis index; f-i liver levels of TC: total cholesterol (f); FC: free cholesterol (g); CE: cholesterol ester (h); TG: triglycerides (i). Different letters indicated significant difference at $\mathrm{P}<0.05$. 

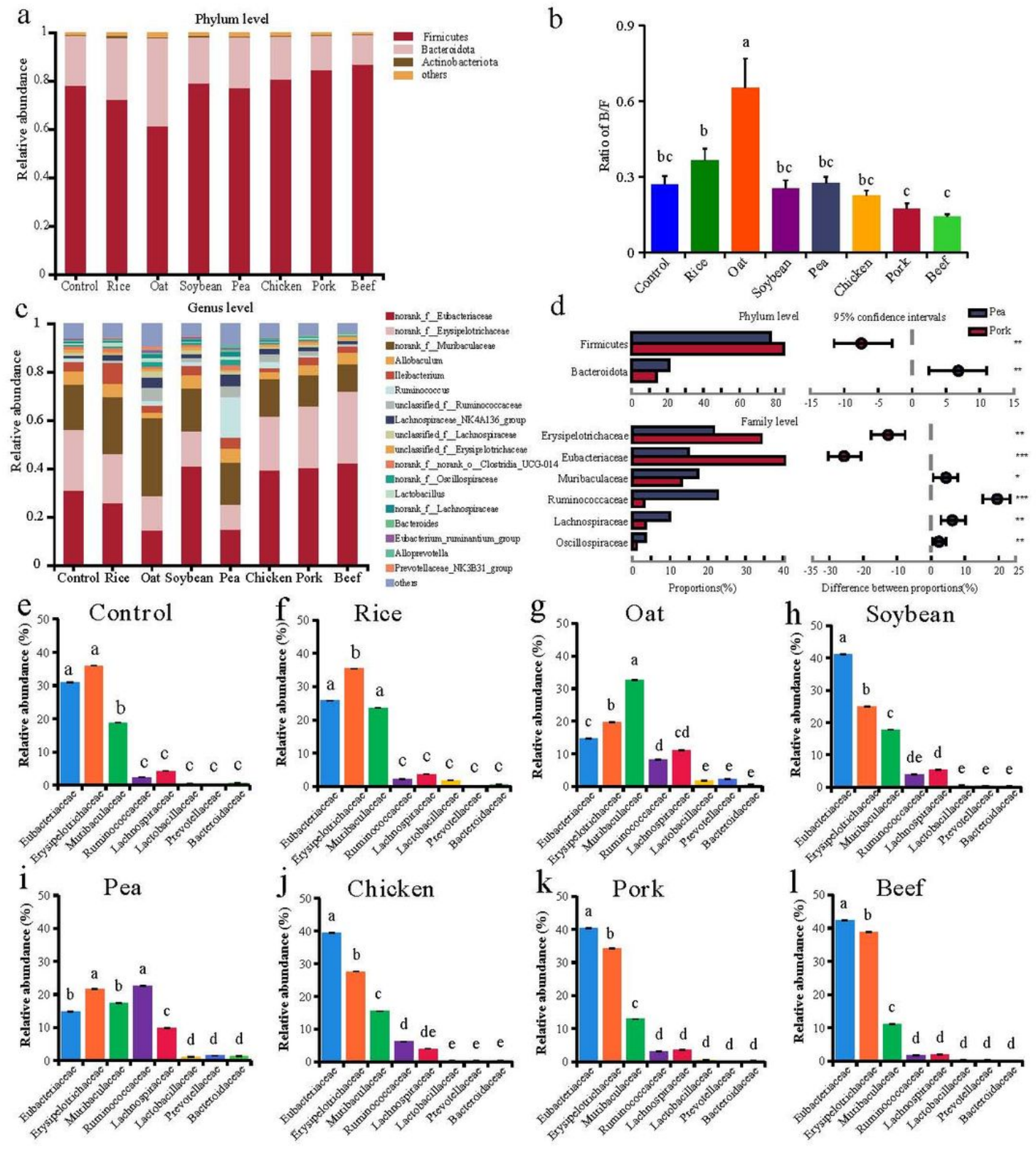

Figure 2

Changes of gut microbiota composition in response to diverse dietary proteins. a The composition of gut microbiota in fecal samples from hamsters after 30 days administration of diverse proteins at phylum level; $b$ The ratio of Bacteroidota and Firmicutes in different groups. c The composition of gut microbiota in fecal samples from hamsters after 30 days administration of diverse proteins at genus level; taxa with abundance below $1 \%$ was presented as others. $d$ Gut microbiota significantly different between pea and pork protein groups at phylum and family level. The bar plots show the abundance of diverse bacterial. Positive differences in mean of relative abundance indicate bacterial with higher abundance in the pea protein group, while negative differences indicate bacterial with higher abundance in pork protein group. Statistical analysis was performed by the Wilcoxon test coupling with fdr correction. e-I Discrepant bacteria at family level in Control (e), Rice (f), Oat (g), Soybean (h), Pea (i), Chicken (j), Pork (k), Beef (I). Different letters indicated significant difference at $P<0.05 .{ }^{*} P<0.05,{ }^{\star *} P<0.01 * \star \star P<0.001$. 


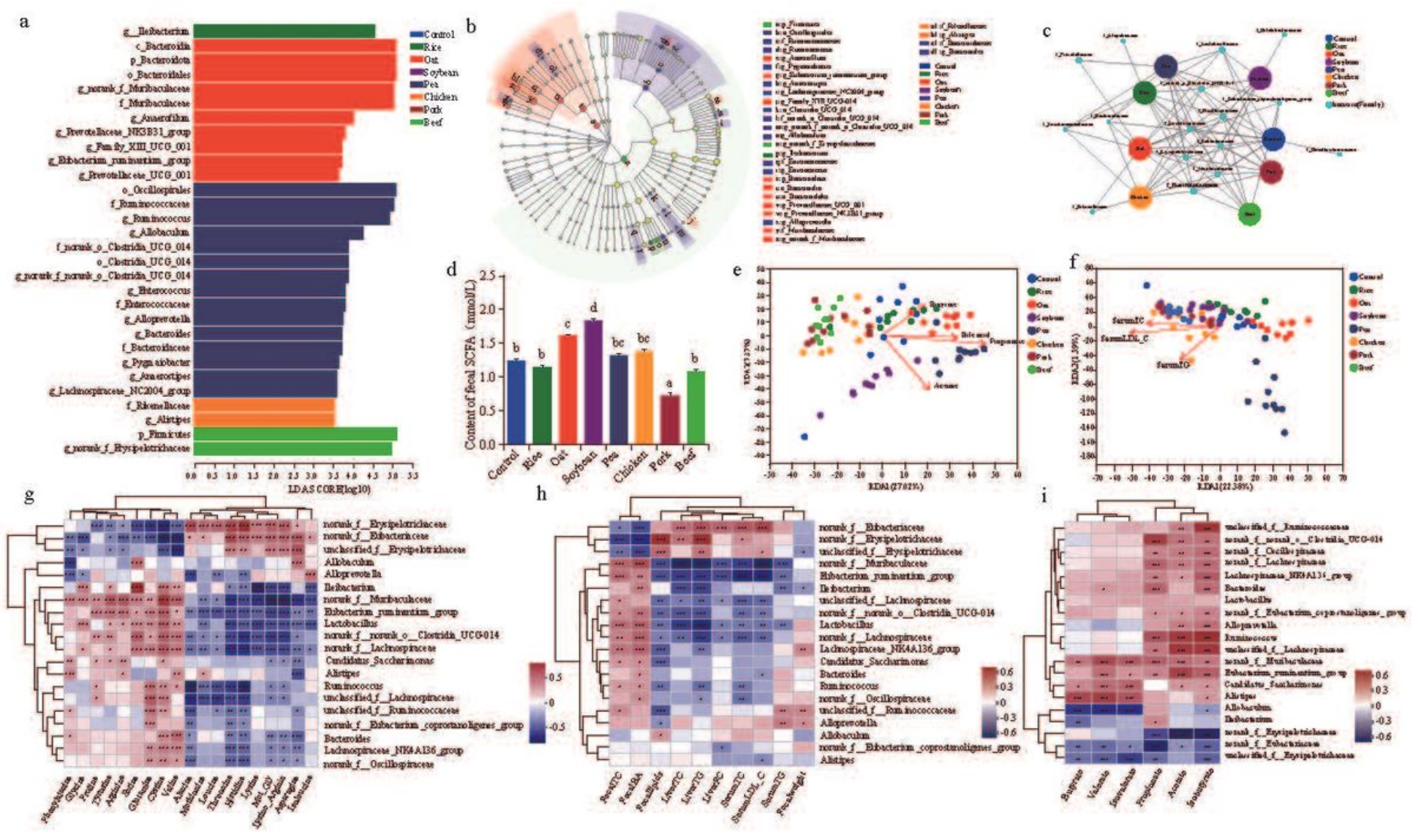

Figure 3

Discrepant bacteria and correlation analysis. a Taxa with linear discirminant analysis (LDA) score 3 or greater from phylum to genus levels in gut microbiota communities of diverse protein groups. b Taxa with linear discirminant analysis effect size (LEfSe) representing bacteria from genus to phylum level in different groups c The interconnection networks of gut microbiota at family level in different groups. Species node sizes represent relative abundance at family levels and edges represent the association patterns of individual family with the dietary protein types. d Contents of short chain fatty acids (SCFAs) in different groups. Redundancy analysis (RDA) of samples from different groups based on OTU data and gut microbial metabolites (e); serum lipid profile (f). Correction analysis of top 20 genera and amino acid (AA) composition of diverse proteins (g); lipid profiles (h); gut microbial metabolites (i). Different letters indicated significant difference at $P<0.05$. ${ }^{\star} P<0.05, \star \star P<0.01 * \star \star P<0.001$. 


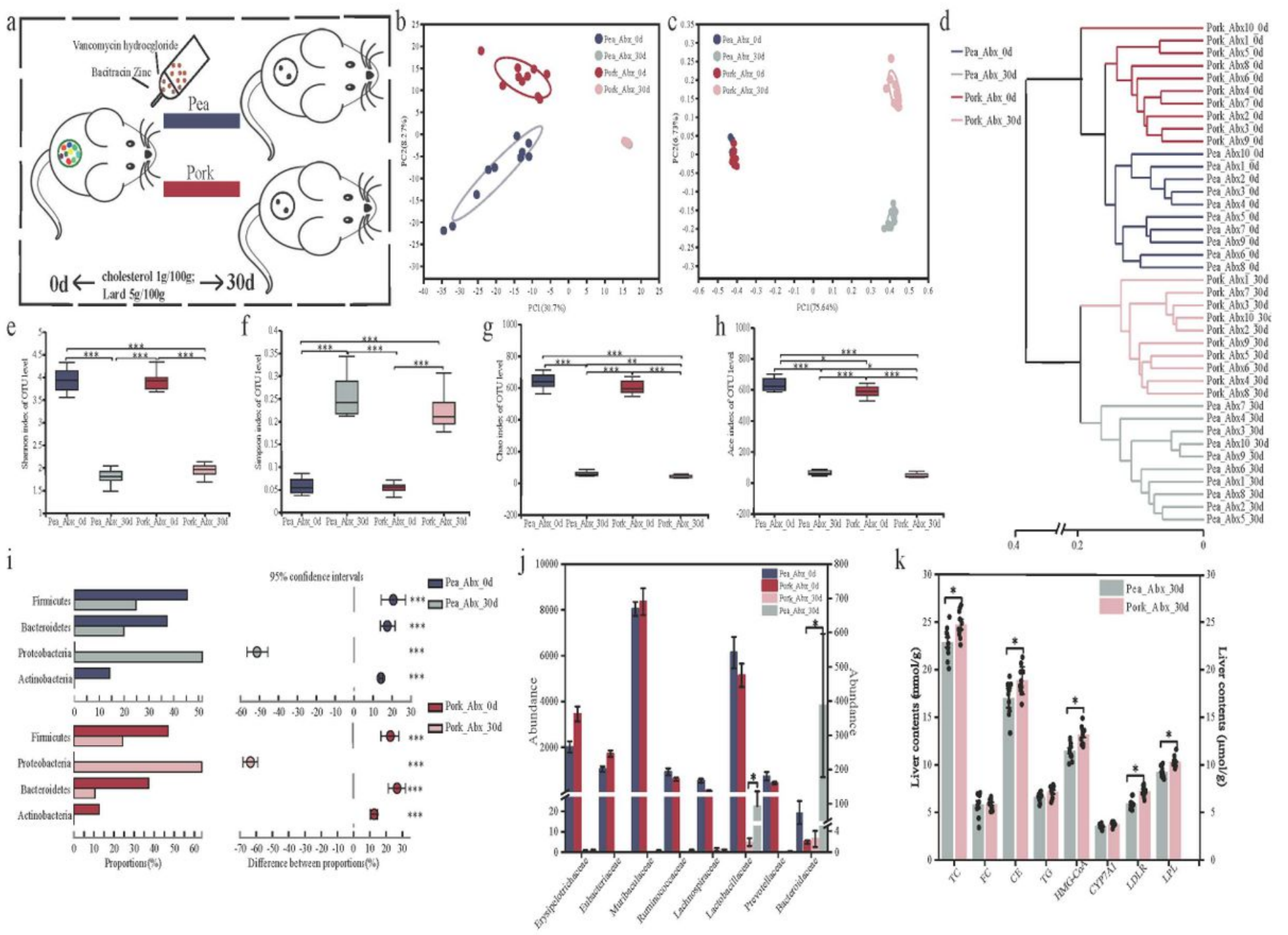

Figure 4

Effects of pea and pork protein with antibiotics treatment on gut microbiota composition and structure. a The overview of the antibiotics study design. b Principal coordinate analysis (PCA). c Principal coordinate analysis (PCoA) based on unweighted UniFrac of all groups at $0 \mathrm{~d}$ and $30 \mathrm{~d}$. $\mathrm{d}$ Hierarchial clustering of gut bacteria at OTU level. e-h Boxplot of alpha diversity indices at OTU level expressed as Shannon index (e); Simpson index (f); Chao index (g); Ace index (h). i Mean proportions of discrepant species at phylum level in different groups by two-group comparisons using Welchs't-test: Pea_Abx_0d vs. Pea_Abx_30d; Pork_Abx_0d vs. Pork_Abx_30d. j Abundance of the discrepant gut microbiota at family level. k liver contents of TC: total cholesterol; FC: free cholesterol; CE: cholesterol ester; TG: triglycerides; HMG-CoA: Hydroxymethyl glutarate monoacyl coa reductase; CYP7A1: cholesterol 7-alpha-hydroxylase; LDLR: low density lipoprotein receptor; LPL: lipoprotein lipase. *P $<0.05$, **P $<$ $0.01 * * * \mathrm{P}<0.001$. 


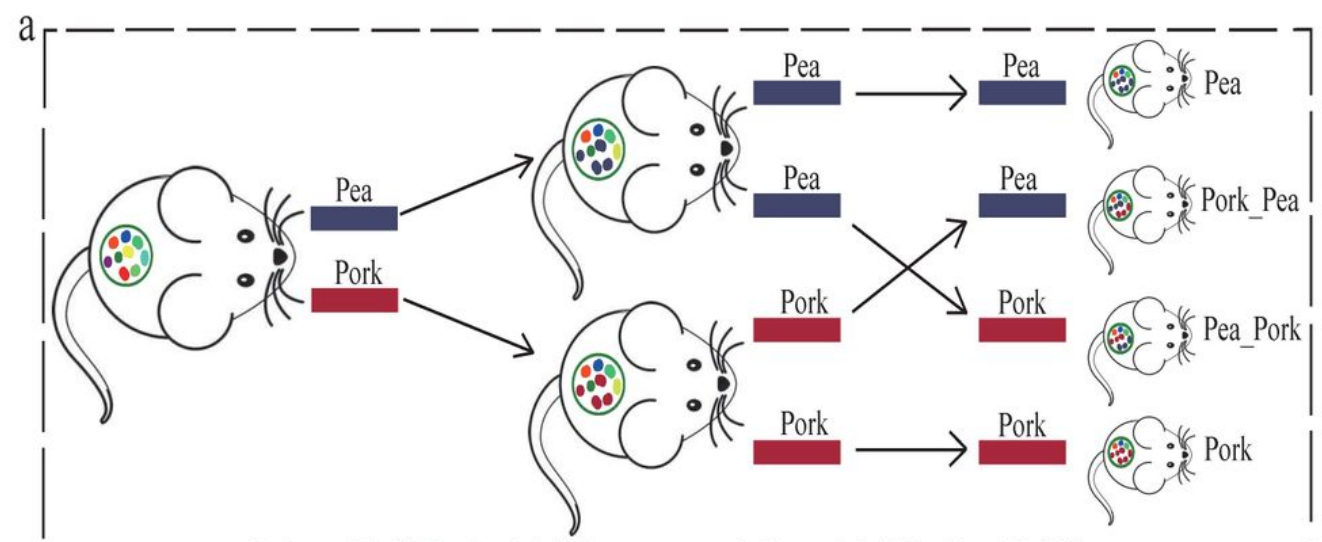

0 day $\stackrel{\text { cholesterol } 1 \mathrm{~g} / 100 \mathrm{~g} ; \text { Lard } 5 \mathrm{~g} / 100 \mathrm{~g}}{\longrightarrow} 30$ ty day $\stackrel{\text { cholesterol } 1 \mathrm{~g} / 100 \mathrm{~g} ; \text { Lard } 5 \mathrm{~g} / 100 \mathrm{~g}}{>} 60$ ty day
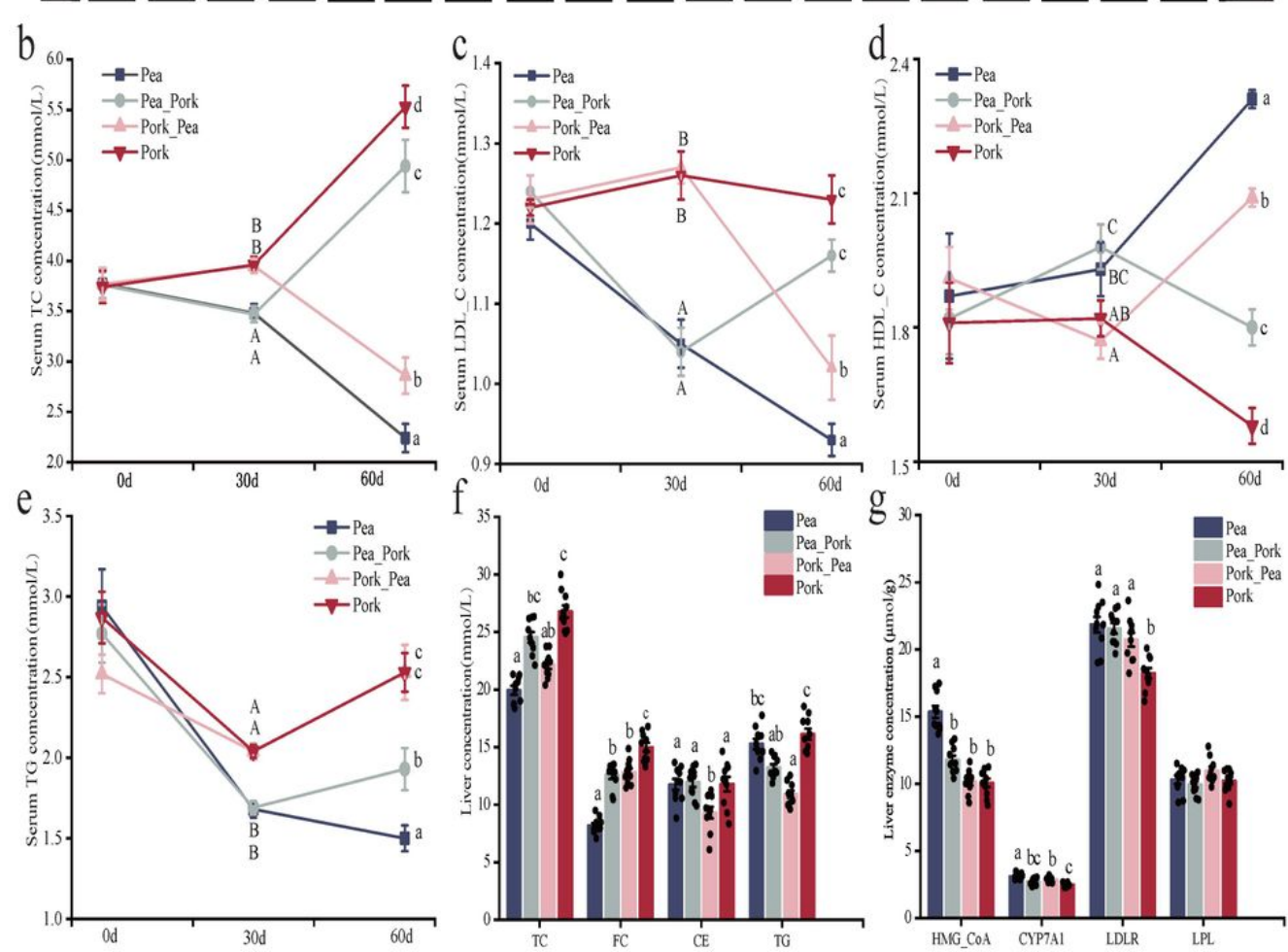

Figure 5

Effects of pea and pork protein supplement on cholesterol level in hamsters. a The overview of the cross-over intervention of pea and pork protein study design. b-e Serum levels of TC: total cholesterol (b); LDL-C: low density lipoprotein cholesterol (c); HDL-C: high density lipoprotein cholesterol (d); TG: triglycerides (e); f liver concentrations of TC: total cholesterol; FC: free cholesterol; CE: cholesterol ester; TG: triglycerides. g liver concentrations of HMG-CoA: Hydroxymethyl glutarate monoacyl coa reductase; CYP7A1: cholesterol 7-alpha-hydroxylase; LDLR: Iow density lipoprotein receptor; LPL: lipoprotein lipase. Different letters indicated significant difference at $p<0.05$. 

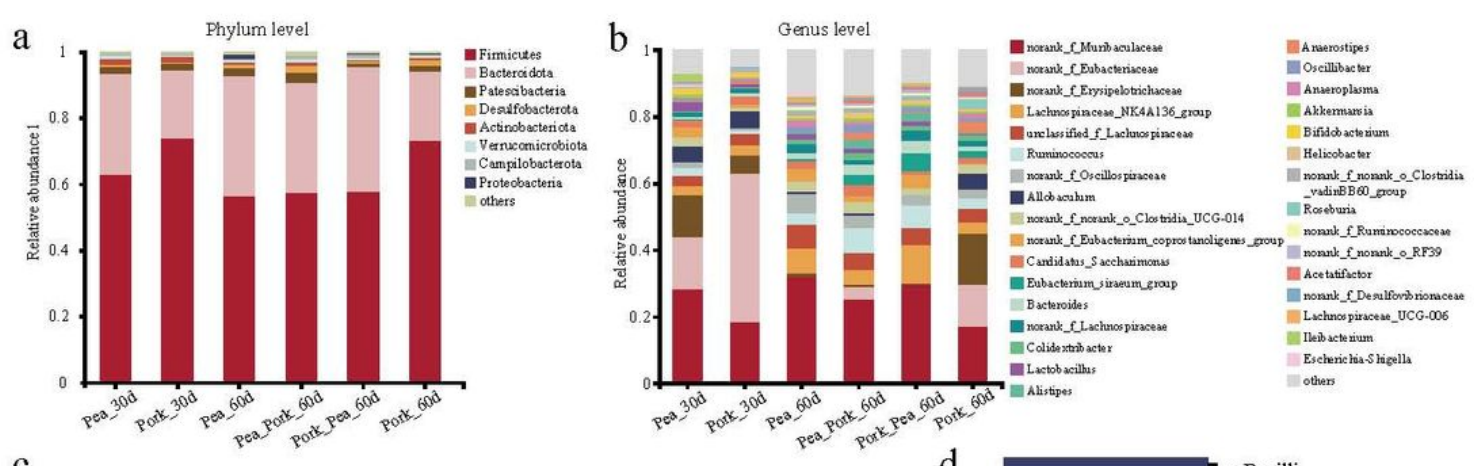

C
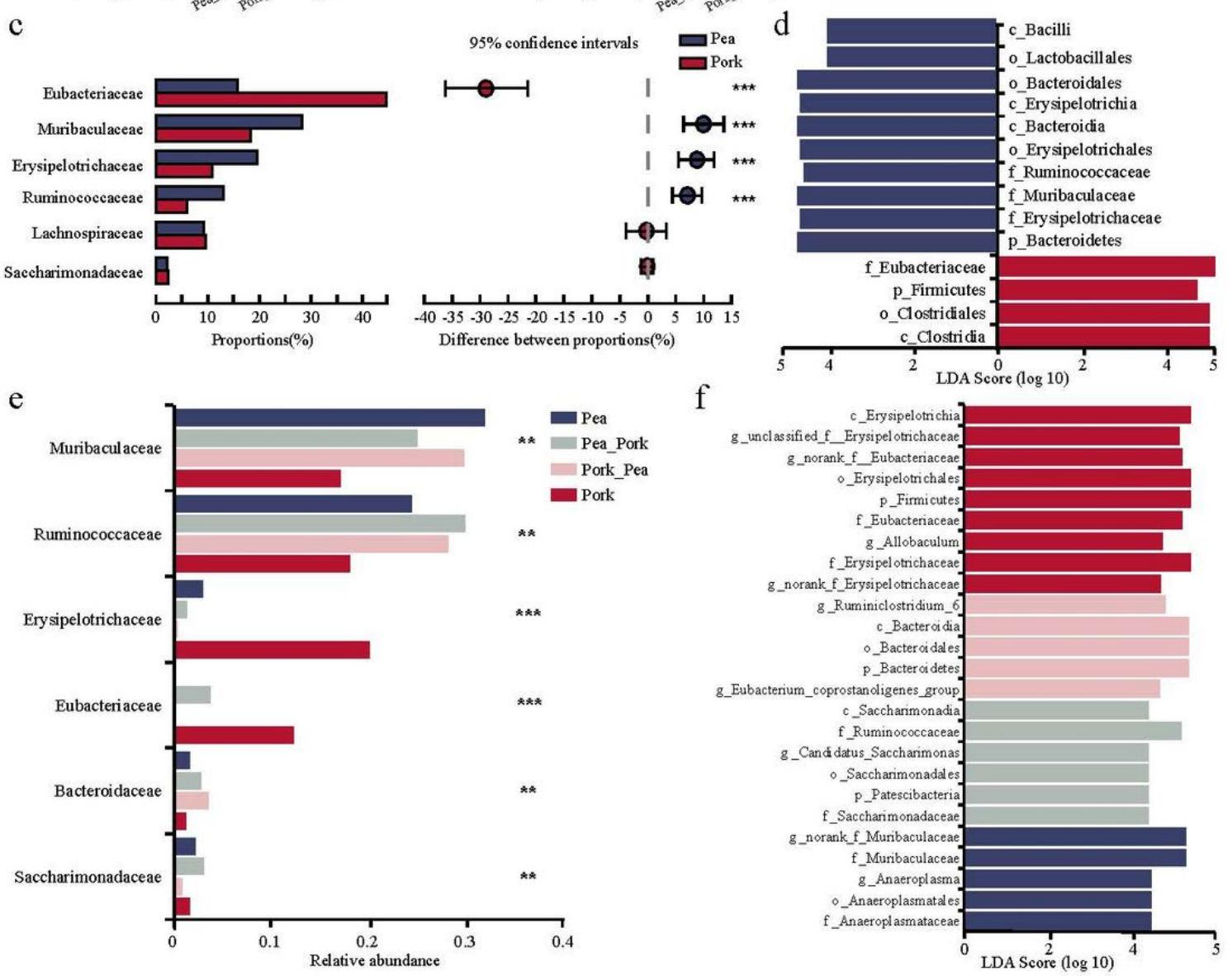

Figure 6

Changes of gut microbiota composition in response to pea protein and pork protein. a-b The composition of gut microbiota at phylum level (a) or genus level (b) in different groups; taxa with abundance below $1 \%$ was presented as others. c Mean proportions of discrepant species on phylum level in different groups at 30d by two-group comparisons using Welchs't-test: Pea vs. Pork at $30 \mathrm{~d}$. $\mathrm{d}$ Taxa with LDA score 3 or greater from phylum to genus levels in gut microbiota communities of pea and pork protein groups at $30 \mathrm{~d}$. e Proportions of gut microbiota on family level with significant difference among different groups at $60 \mathrm{~d}$. Kruskal-Wallis $\mathrm{H}$ test coupling with False Discovery Rate (fdr) correction was applied to evaluate the significant difference among the four groups at $60 \mathrm{~d}$. $f$ Taxa with LDA score 3 or greater from phylum to genus levels in gut microbiota communities of different groups at $60 \mathrm{~d}$. ${ }^{*} \mathrm{P}<$ $0.05, * * \mathrm{P}<0.01 * * * \mathrm{P}<0.001$. 
a

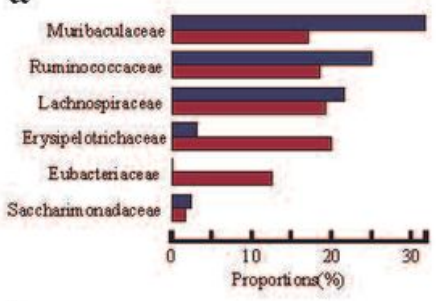

$\mathrm{c}$

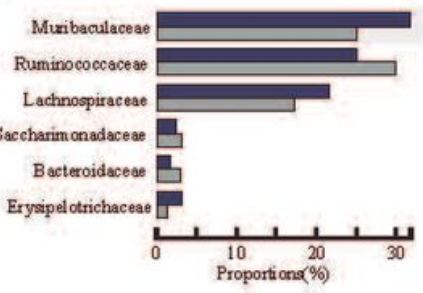

e

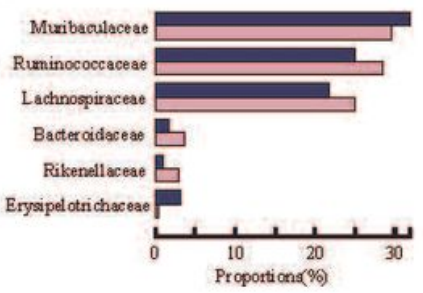

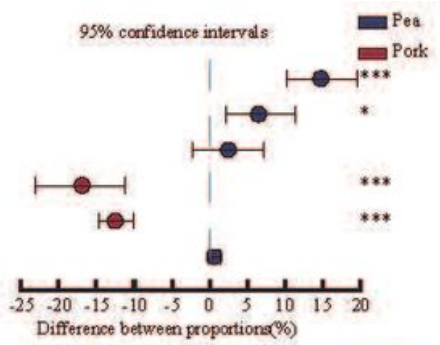

Difference between proportions $(\%)$

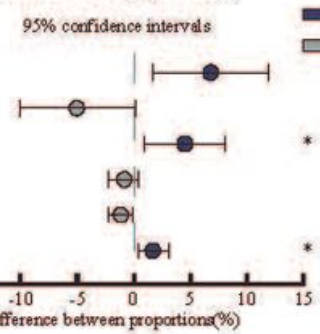

Pea $\square$ Pea_Pork

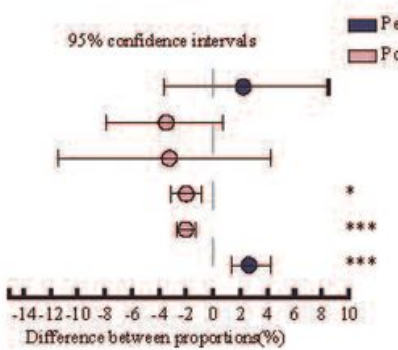

d

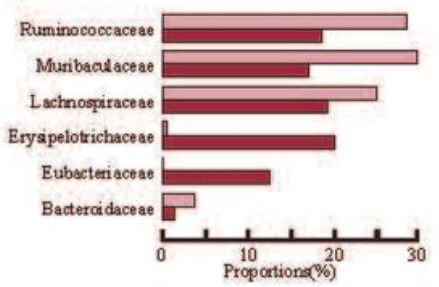

$\mathrm{b}$
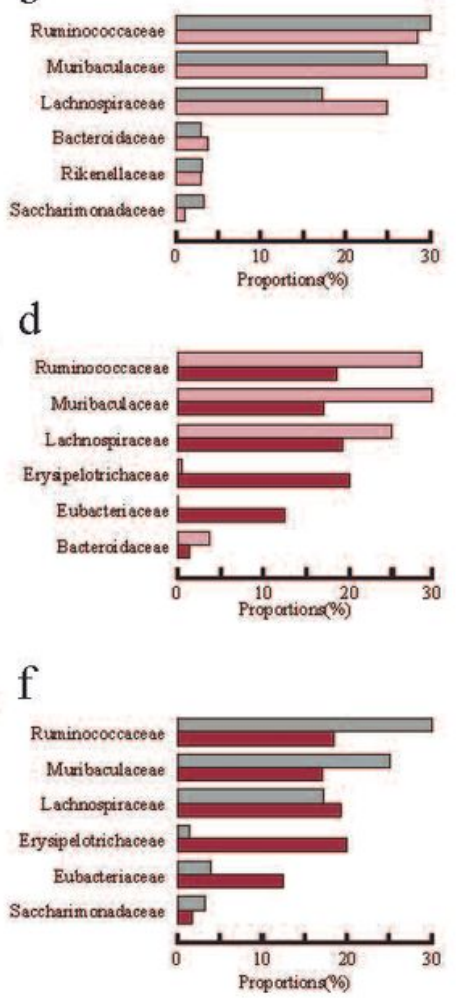

95\% confidence intervals $\square$ Pea_Pork $\square$ Pork_Pea

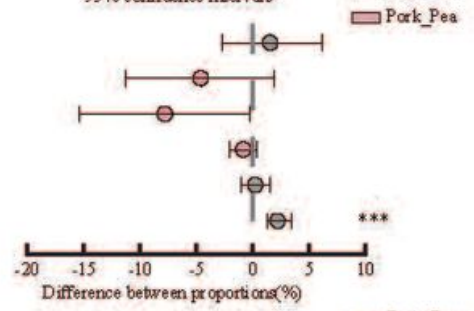

$95 \%$ confidence intervals $\square$ Pork_Pea
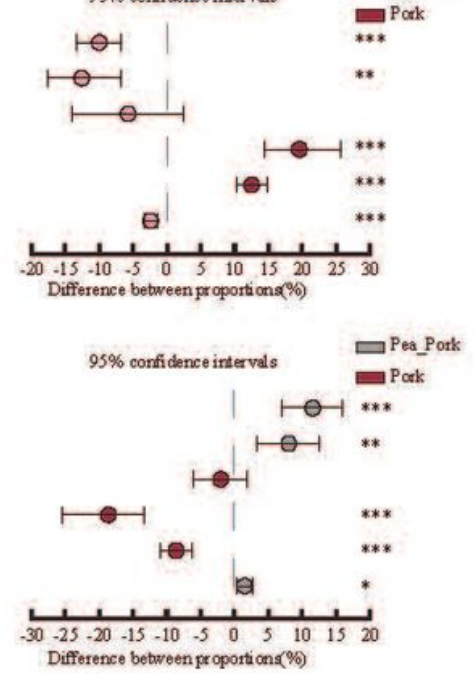

\section{Figure 7}

Mean proportions of discrepant species at phylum level in different groups at $60 \mathrm{~d}$ by two-group comparisons using Welchs't-test: a$\mathrm{f}$ Pea vs. Pork (a); Pea_Pork vs. Pork_Pea (b); Pea vs. Pea_Pork (c); Pork_Pea vs. Pork (d); Pea vs. Pork_Pea (e); Pea_Pork vs. Pork (f). *P $<0.05,{ }^{\star} * \mathrm{P}<0.01 * \star \star P<0.001$. 


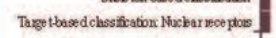

Sigral tareduction Sinnaing moke cules and intenctorn

Foling, scoting ard dgyadation

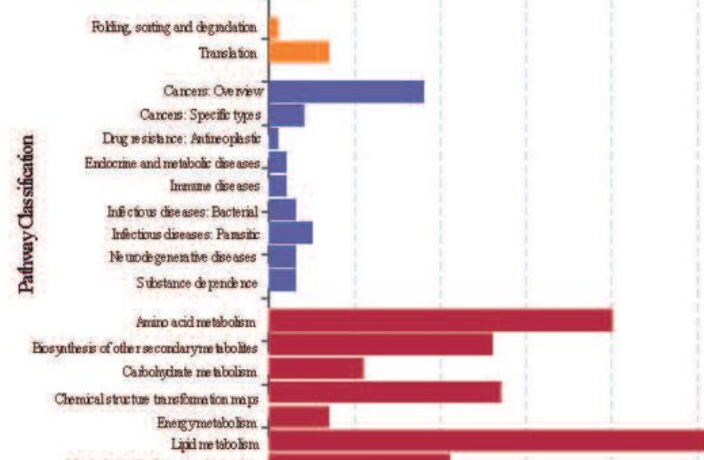

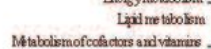

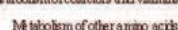

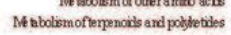

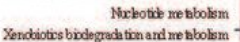
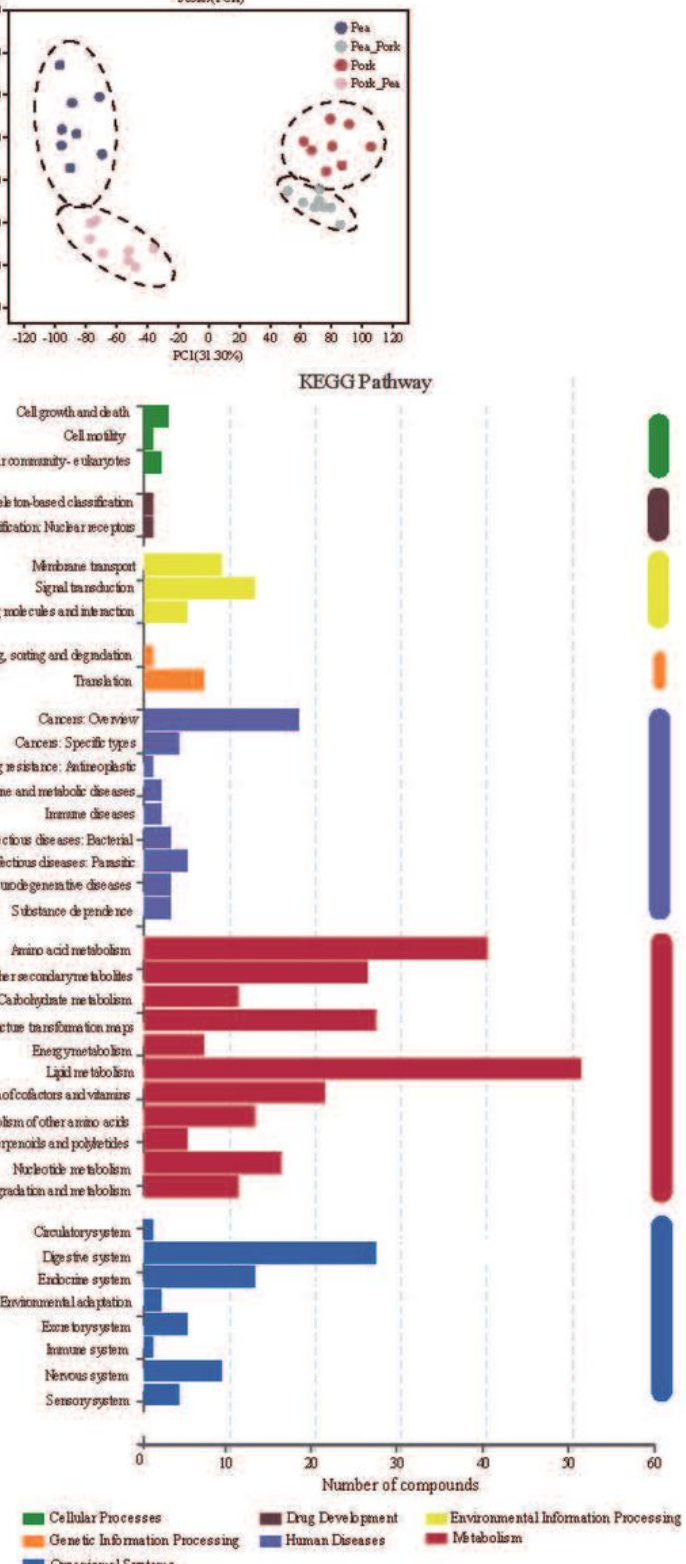
KEGG Pathway a

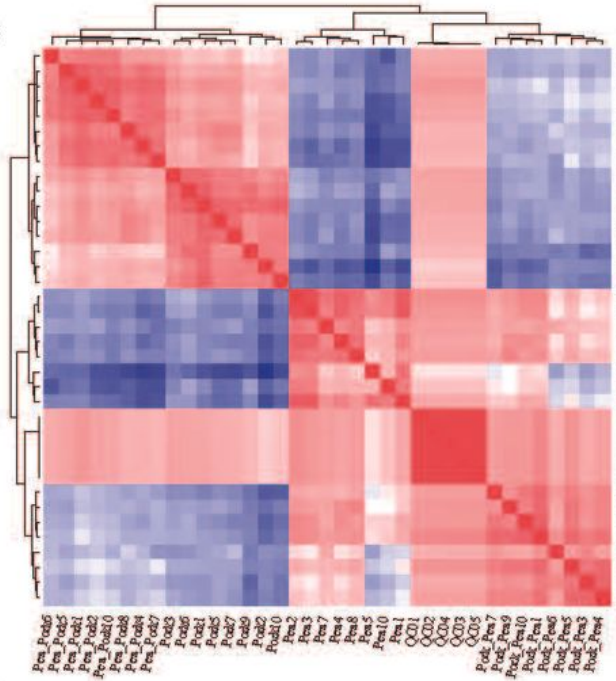

c

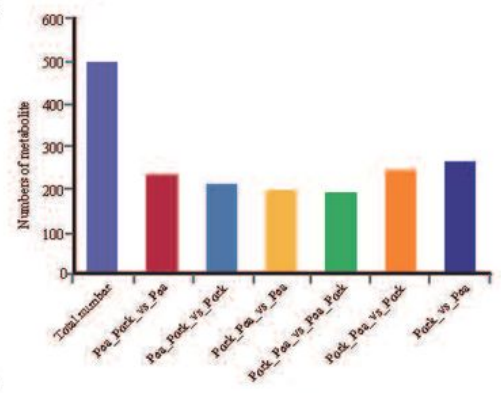

d

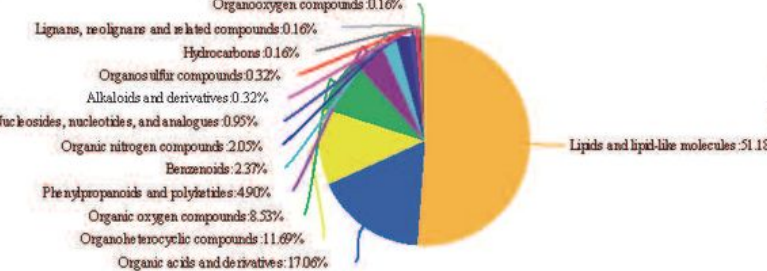

Organic acile and de nathes: $1700 \%$

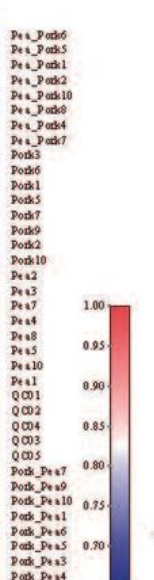

$\operatorname{soses}(\mathrm{PCA})$

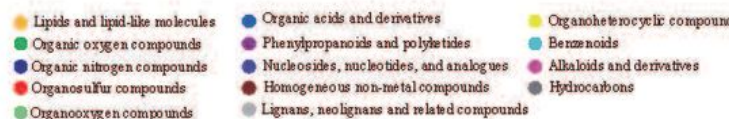

- Allaloids and derivatives s

Figure 8

Overview of metabolic signatures among all groups. a Heat map showing pairwise comparisons of different samples based on the detected metabolites. b PCA of metabolic features among all groups. c Numbers of discrepant metabolites in comparison of the two groups. d Pie chart based on counts of HMDB chemical taxonomy (superclass) for all metabolites detected in this study class. e KEGG pathway classification of metabolites detected and annotated. 

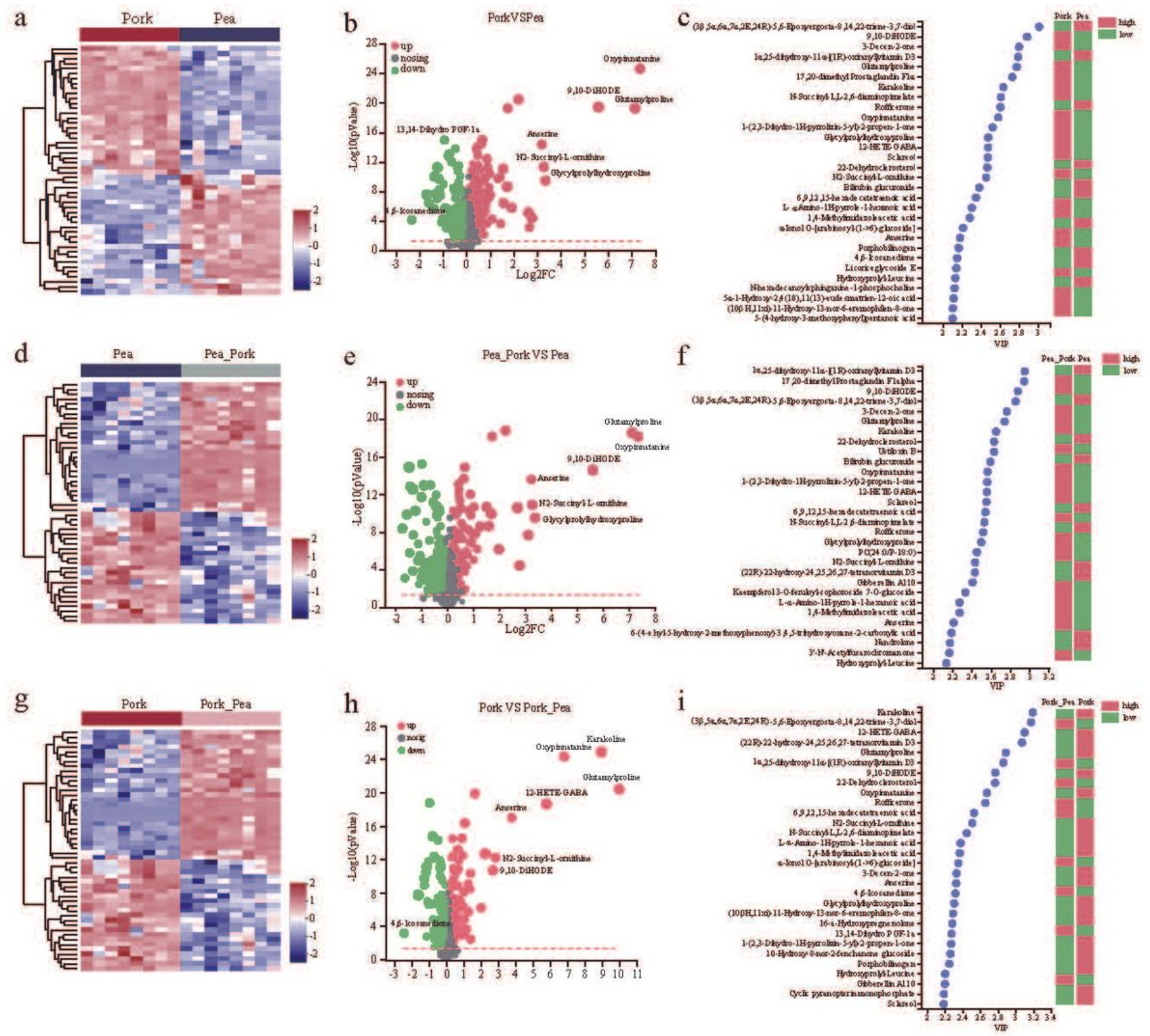

Figure 9

Cecal metabolome alterations. a-c Comparision between pork and pea groups by heatmap of discrepant metabolites (a); volcano plot of discrepant metabolites (b); discrepant metabolic signatures ranked by variable importance in projection (VIP) score (c). $d$-f Comparision between Pea_Pork and pea groups by heatmap of discrepant metabolites (d); volcano plot of discrepant metabolites (e); discrepant metabolic signatures ranked by VIP score (f). g-i Comparision between Pork_Pea and pork groups by heatmap of discrepant metabolites (g); volcano plot of discrepant metabolites (h); discrepant metabolic signatures ranked by VIP score (i). The red spots or pieces indicate upregulation, and the green ones indicate downregulation. 


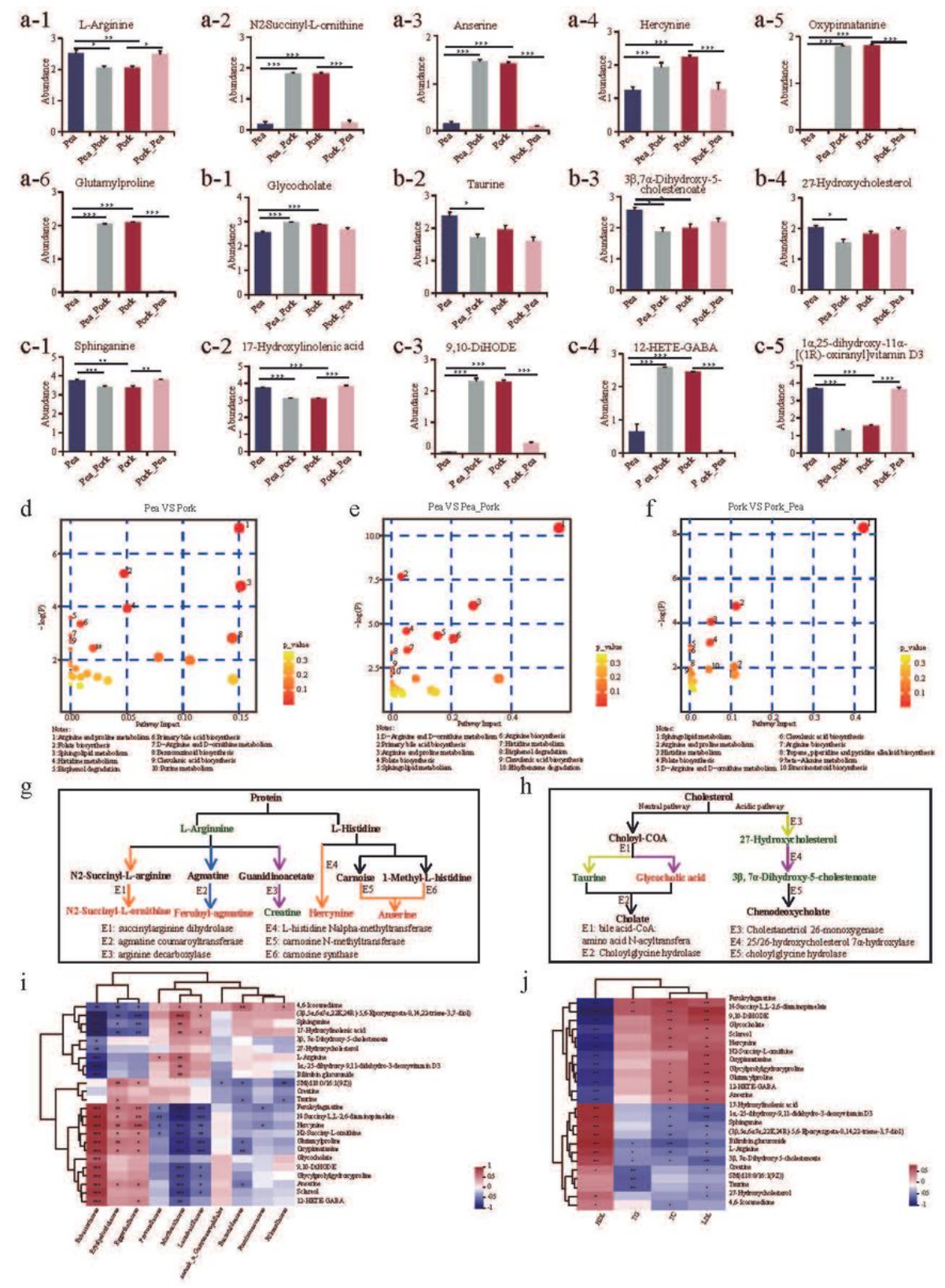

Figure 10

Alterations of specific cecal metabolites and pathways. Relative expression of discrepant metabolites: L-Arginine (a-1), N2Succinyl-L-ornithine (a-2), Anserine (a-3), Hercynine (a-4), Oxypinnatanine (a-5), Glutamylproline (a-6), Glycocholate (b-1), Taurine (b-2), 3ß,7a-Dihudroxy-5-cholestenoate (b-3), 27-Hydroxycholesterol (b-4), Sphinganine (c-1), 17-Hydroxylinolenic acid (c-2), 9,10DiHODE (c-3), 12-HETE-GABA (c-4), 1a,25-Dihydroxy-11alpha-[(1R)-oxiranyl]vitaminD3 (c-5). d-f The main metabolic pathways according to integrative analysis of the pathway impact and $P$ value of metabolic signatures in comparison of pork and pea (d), Pea_Pork and pea (e), pork and Pork_Pea (f).g A diagram of major relevant pathway of arginine and histidine metabolism. Green color of metabolites represents the up-regulation and red color represents the down-regulation in the presence of pea protein. Arrow with different color represents significantly different pathway observed in comparison of different groups: orange represents pork vs. pea, Pea_Pork vs. pea, pork vs. Pork_Pea; purple represents pork vs. pea, Pea_Pork vs. pea; blue represents pork vs. pea, pork vs. pork_Pea. h A diagram major relevant pathway of primary bile acid biosynthesis. Green and red metabolites represent up-regulation and down-regulation in the presence of pea protein. Arrow with different color represents significantly different pathway observed in comparison of different groups: purple represents pork vs. pea, Pea_Pork vs. pea; yellow represents Pea_Pork vs. pea, respectively. i Correlation analysis of specific metabolites with gut microbiota at family level. j Correlation analysis of specific metabolites with serum lipid profiles. 


\section{Supplementary Files}

This is a list of supplementary files associated with this preprint. Click to download.

- Additionalfile1.pdf 